\title{
Synthesis of 3-deoxy-2-uloses via the indium-mediated allylation reaction
}

\author{
Manuel Gintner ${ }^{1}$ (1) Christian Denner ${ }^{1} \cdot$ Christoph Schmölzer $^{1} \cdot$ Michael Fischer $^{1} \cdot$ Peter Frühauf $^{2}$. \\ Hanspeter Kählig ${ }^{1} \cdot$ Walther Schmid ${ }^{1}$
}

Received: 21 March 2019 / Accepted: 20 April 2019 / Published online: 2 May 2019

(c) The Author(s) 2019

\begin{abstract}
We utilized the indium-mediated allylation reaction for the synthesis of carbohydrate structures containing the 3-deoxy2-ulose motif, a barely investigated compound class. The stereoselective outcome can be controlled by the presence or absence of a chelating group in $\alpha$-position to the carbonyl function. By introduction of an UV-active allyl building block, we enabled epimer separation by HPLC towards the synthesis of 3-deoxy-D-glycero-D-galacto-2-nonulose, the carboxylreduced analogue of widely distributed 3-deoxy-D-glycero-D-galacto-nonulosonic acid (Kdn). Ozonolysis of the introduced 2-C-methylidenepropan-1-ol motif provided the desired 3-deoxy-2-uloses.
\end{abstract}

\section{Graphical abstract}

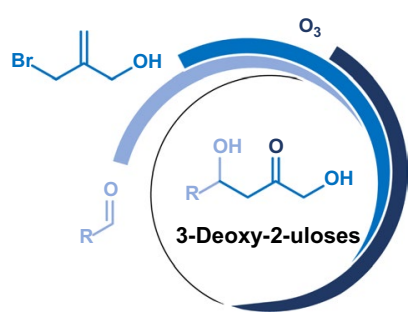

Keywords Carbohydrates · 3-Deoxy-2-uloses $\cdot$ Indium-mediated allylation $\cdot$ Organometallic compounds $\cdot$ Ozonolysis

Dedicated to Professor Dr. Heinz Falk on the happy occasion of his 80th birthday anniversary.

Walther Schmid: deceased, Dec 2017.

Electronic supplementary material The online version of this article (https://doi.org/10.1007/s00706-019-02438-y) contains supplementary material, which is available to authorized users.

Manuel Gintner

manuel.gintner@univie.ac.at

1 Institute of Organic Chemistry, University of Vienna, Vienna, Austria

2 Institute of Analytical Chemistry, University of Vienna, Vienna, Austria

\section{Introduction}

The indium-mediated allylation reaction has proven to be an efficient and reliable $\mathrm{C}-\mathrm{C}$ bond formation method offering many advantages over traditional organometallic compounds [1-3]. Typically, it allows for high regio-, enantio-, as well as diastereoselectivity and tolerates a wide range of functional groups [4-7]. Moreover, indium-mediated allylations can be performed in water, rendering the possibility to fully avoid the use of dry and flammable solvents. This allows for safer and environmentally friendly chemistry, satisfying the demands of green chemistry [1-4]. The reactions generally proceed under mild conditions at room temperature without any promoter, in contrast to the use of other metals such as zinc or tin, which requires acid catalysis, elevated temperatures or sonication, often leading to increasing amounts of undesired side products $[8,9]$. Because of 


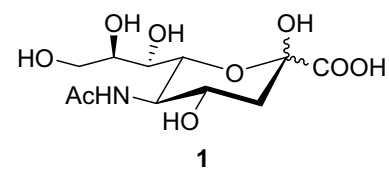<smiles>O=C(O)[C@]1(O)C[C@@H](O)[C@@H](O)[C@H](C(O)CO)O1</smiles>
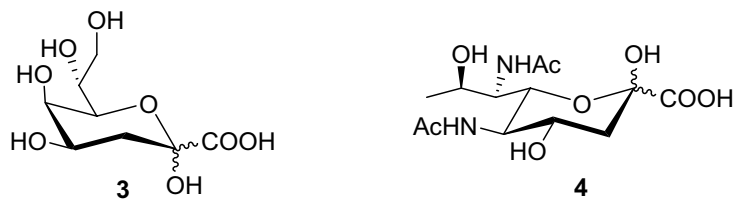

Fig. 1 Natural products (Neu5Ac, 1), (Kdn, 2), (Kdo, 3), (Leg, 4) synthesized via an indium-mediated allylation reaction

\section{Scheme 1}

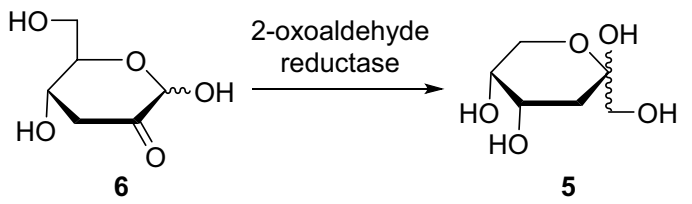

its superior reactivity, the indium-mediated allylation has been applied in numerous natural product syntheses by us and others, e.g. $N$-acetylneuraminic acid (Neu5Ac, 1) [10, 11], 3-deoxy-D-glycero-D-galacto-nonulosonic acid (Kdn, 2) [12], 3-deoxy-D-manno-2-octulosonic acid (Kdo, 3) [13] or legionaminic acid (Leg, 4) [14, 15] (Fig. 1).

An intriguing compound class that has hardly been addressed by organic synthesis is the 3-deoxy-2-ulose representing an interesting and rare subcategory of the ketose family. Being the $\mathrm{C}-1$ reduced analogue of naturally essential ulosonic acids, these structures have potential biological importance and therefore are attractive target molecules [16]. A naturally occurring representative is 3-deoxy-D-erythro-2-hexulose (3-deoxy-D-fructose, 5), which has been found in mammalian blood and urine [17-19] in concentrations that are elevated in diabetes [20]. As degradation product, it arises from 3-deoxyglucosone (6), a key intermediate in the nonenzymatic polymerization and browning of proteins by glucose (Maillard reaction), which is also formed in vivo by $\mathrm{D}$-fructose [21], D-fructose-3-phosphate [22], or Amadori adducts to proteins [19]. In mammals, a significant proportion of 3-deoxyglucosone (6) is reduced to 3-deoxy-D-erythro2-hexulose (5) presumably to decrease its reactivity, thus being less damaging to tissues (Scheme 1) [23].

An earlier approach of our group regarding the synthesis of 3-deoxy-2-uloses utilized the indium-mediated allylation reaction employing allyl bromide for the elongation of different aldoses, such as $N$-acetyl-D-mannosamine or D-erythrose (Scheme 2). The terminal allyl moiety of $\mathbf{8}$ was subsequently oxidized using potassium permanganate under acidic conditions yielding the desired 3-deoxy-2-ulose functionality in 9. However, the acidity of the 3-deoxy position prohibited standard de- $O$-acetylation methods as elimination product $\mathbf{1 0}$ was formed exclusively. Hence, a thioketal moiety was introduced at position $\mathrm{C}-2$ to give $\mathbf{1 1}$ and enabled de- $O$-acetylation to 12. Final removal of the thioketal yielded 3-deoxy-2-ulose 13 [24].

Scheme 2

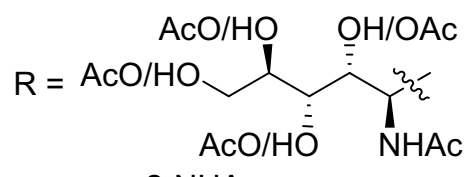
2-NHAc-D-manno

1) allyl bromide, In

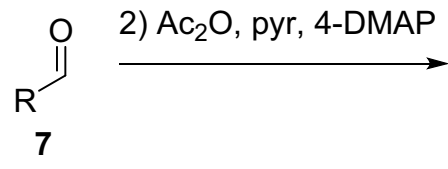<smiles>[R]C(CC=C)OC(C)=O</smiles><smiles>[R]C(O)CC(=O)CO</smiles><smiles>[Pb]=[Pb]</smiles>

13
8<smiles>[R]O[13C](=O)[O-]</smiles><smiles>[R]C=CC(=O)CO</smiles><smiles>[R]C([14CH3])CC(=O)CO</smiles>
$\mathrm{EtSH}, \mathrm{SnCl}_{2} \cdot 2 \mathrm{H}_{2} \mathrm{O}$, $\mathrm{CH}_{2} \mathrm{Cl}_{2}$, microwave 
Herein, we report a novel approach towards 3-deoxy2-uloses by elongating selected aldoses using 2-(bromomethyl)prop-2-en-1-ol (14) in an indium-mediated allylation reaction. The presence or absence of a chelating group next to the carbonyl function directs the formation of the corresponding syn- or anti-product respectively, as we verify during the synthesis of 3-deoxy-D-erythro-2-hexulose (5) and 3-deoxy-D-gluco-2-octulose (15). Thus, introduction of an appropriate protecting group in $\alpha$-position provides diastereoselective control. A benzyl protected allyl building block (2-(benzyloxymethyl)allyl bromide, 16) can be employed to enable UV detection for epimer separation by HPLC of the epimeric mixture obtained after the elongation reaction.

\section{Results and discussions}

The indium-mediated allylation using 2-(bromomethyl)prop-2-en-1-ol (14) was applied on three different aldoses-2,3-O-isopropylidene-D-glyceraldehyde (17), D-arabinose (18), and D-mannose (19)_introducing a 2-C-methylidenepropan-1-ol motif. Allyl component 14 was synthesized starting from triethyl phosphonoacetate (20) performing a known Horner-Wadsworth-Emmons protocol [25], followed by bromination [25] and DIBALH-reduction [26]. Due to the volatility of the allyl building block 14, the yield for its preparation is modest. After allylation, the generated epimeric mixtures could be separated by conventional silica gel chromatography, when employing 2,3-O-isopropylidene-D-glyceraldehyde (17) and D-arabinose (18) as starting materials. However, for the separation of the epimers obtained from the elongation of D-mannose (19), HPLC had to be performed. To enable UV-detection during HPLC, a benzyl protected allyl building block 16 has been used. Final ozonolysis of the elongated aldoses provided the desired 3-deoxy-2-uloses in good to excellent yields.

2,3-O-Isopropylidene-D-glyceraldehyde (17) has been synthesized from D-mannitol via a known isopropylideneprotection procedure [27] and subsequent oxidative diol cleavage [28]. During the indium-mediated allylation, the absolute configuration of the newly formed stereocenter at position $\mathrm{C}-4$ is influenced by the presence or absence of a chelating group in $\alpha$-position to the reacting carbonyl function. Hence, an isopropylidene-protected hydroxyl function in neighboring position acts as weak chelating group and allows for the preferred formation of the antiproduct (2,3-dideoxy-5,6- $O$-isopropylidene-2- $C$-methylidene-D-erythro-hexitol, anti-21) as demonstrated by the elongation of 2,3-O-isopropylidene-D-glyceraldehyde (17) (Scheme 3). The resulting epimeric mixture was separated by column chromatography. Removal of the isopropylidene group under acidic conditions and subsequent ozonolysis furnished the keto functionality at position C-2. 3-DeoxyD-erythro-2-hexulose (5) was obtained in a yield of $35 \%$ over 3 steps.

The allylation of D-arabinose (18) employing 2-(bromomethyl)prop-2-en-1-ol (14) favored the formation of the corresponding syn-product $(1,4,5,6,7,8$-hexa- $O$-acetyl2,3-dideoxy-2-C-methylidene-D-gluco-octitol, syn-23) due to the strong chelating hydroxyl group in $\alpha$-position to the aldehyde function. Per- $O$-acetylation facilitated epimer separation via column chromatography. Zemplén saponification provided 2,3-dideoxy-2-C-methylidene-D-gluco-octitol (24). The stereochemical relation between the initial $\alpha$-substituent and the newly formed hydroxyl group of compound $\mathbf{2 4}$ has been confirmed by X-ray analysis (Fig. 2). Subsequent ozonolysis generated the keto function at position C-2. 3-DeoxyD-gluco-2-octulose (15) was obtained in a yield of $47 \%$ over 3 steps (Scheme 4).

\section{Scheme 3}

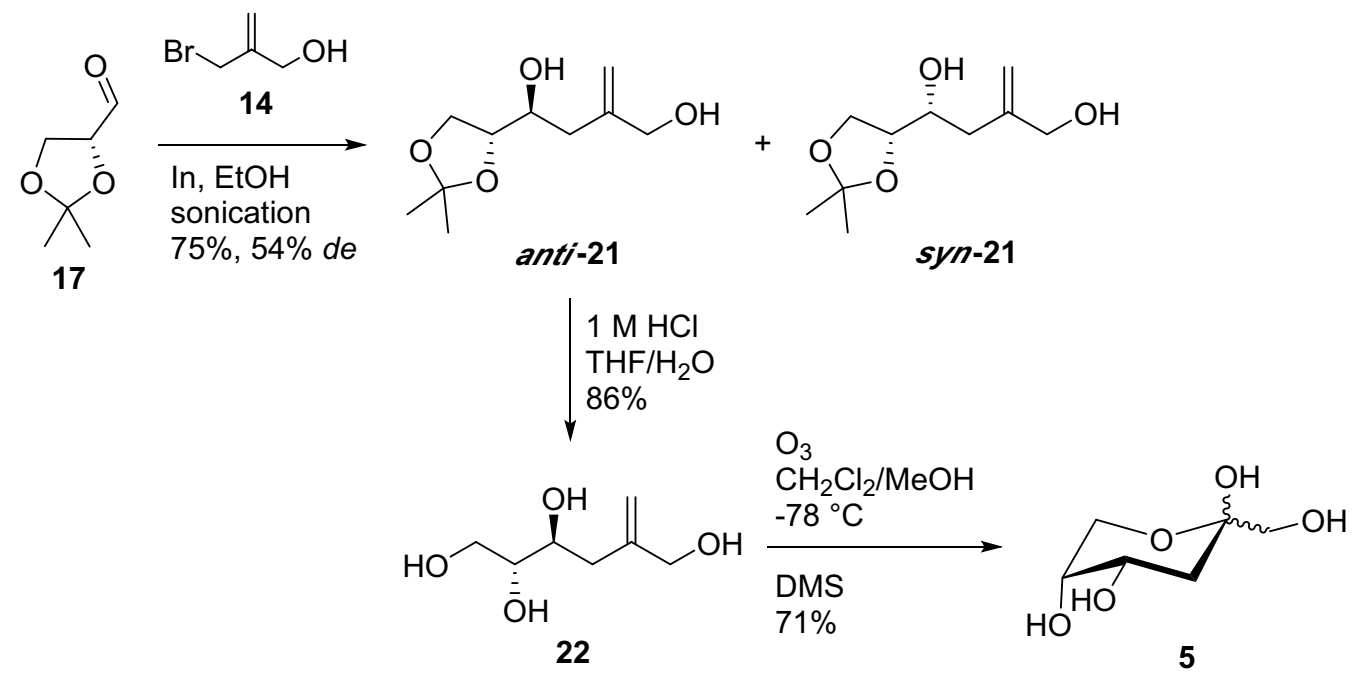




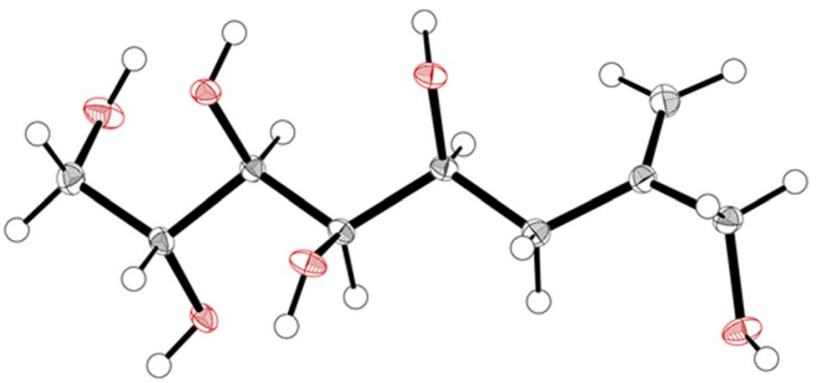

Fig. 2 X-ray analysis of compound 24 confirming its D-gluco-configuration

The elongation of D-mannose (19) utilizing allyl species $\mathbf{1 4}$ preferred the formation of the syn-product $(1,4,5,6,7,8,9$-hepta- $O$-acetyl-2,3-dideoxy-2- $C$-methylidene-D-glycero-D-galacto-nonitol, syn-25) according to
D-arabinose (18). However, separation of the generated epimers in both, their unprotected or per- $O$-acetylated form, could not be accomplished via column chromatography. Also, performing ozonolysis directly after allylation followed by per- $O$-acetylation to compounds syn-/anti-26 did not facilitate isolation of the epimers, but led to an even more complex compound mixture containing epimers, their corresponding anomers as well as side products by elimination, which turned out to be inseparable by conventional column chromatography (Scheme 5).

We decided to tackle this challenge using HPLC. To enable detection via UV-absorbance, we employed 2-(benzyloxymethyl)allyl bromide (16) as allyl species for the elongation in the indium-mediated allylation reaction. Compound 16 was synthesized starting from triethyl phosphonoacetate (20) according to a known Horner-Wadsworth-Emmons protocol [25] to provide ethyl 2-(hydroxymethyl)acrylate (27).

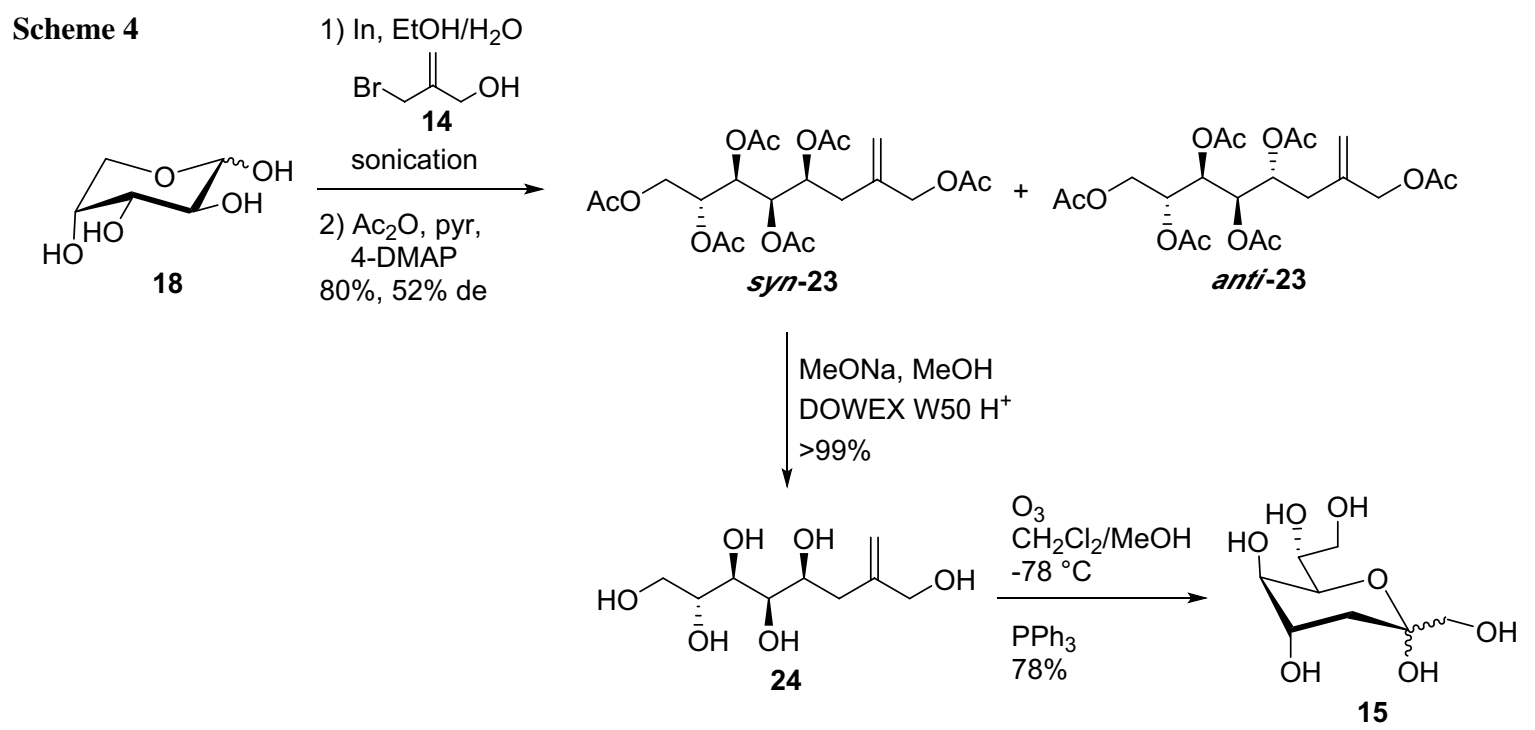

Scheme 5<smiles>OC1C(O)C(O)C(O)C(O)C1O</smiles>

19
1) $\mathrm{In}, \mathrm{EtOH} / \mathrm{H}_{2} \mathrm{O}$<smiles>C=C(O)[14CH2]Br</smiles>

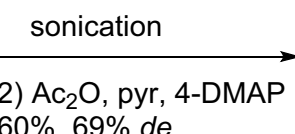
$60 \%, 69 \%$ de

1) In, $\mathrm{EtOH} / \mathrm{H}_{2} \mathrm{O}$<smiles></smiles>

2) $\mathrm{O}_{3}, \mathrm{CH}_{2} \mathrm{Cl}_{2} / \mathrm{MeOH}$ $-78^{\circ} \mathrm{C}$, DMS

3) $\mathrm{Ac}_{2} \mathrm{O}$, pyr, 4-DMAP<smiles>C=C(COC(C)=O)CC(OC(C)=O)C(OC(C)=O)C(OC(C)=O)C(OC(C)=O)C(O)COC(C)=O</smiles>

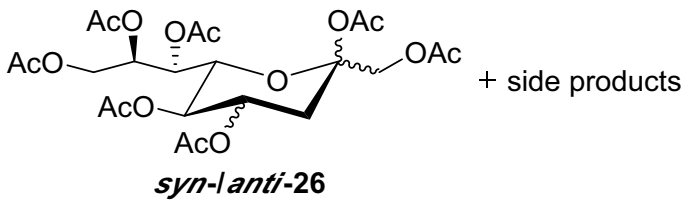


$O$-Benzylation was achieved using benzyl trichloroacetimidate (28) which was synthesized according to a known procedure [29, 30]. Early benzylation in the allyl building block synthesis decreases the volatility of subsequent intermediates, thus facilitating the workup processes. DIBAL-Hreduction and bromination via Appel reaction [31, 32] gave allyl species $\mathbf{1 6}$ in a yield of $37 \%$ over 4 steps (Scheme 6).

Allylation of D-mannose (19) using compound 16 gave a mixture of epimers in a ratio of $4: 1$. Due to the presence of a strong chelating hydroxyl group next to the reacting carbonyl function, we termed the major product syn-31. The epimeric mixture was per- $O$-acetylated and purification via normal-phase HPLC was conducted (Scheme 7). Although separation of the epimers (syn-/anti-31) was accomplished using heptane/ethyl acetate as eluent $(6: 1 \mathrm{v} / \mathrm{v})$, elaborate conditions such as cooling of the column to $15{ }^{\circ} \mathrm{C}$ were necessary to give two separated peaks at retention times of 107 and 112 min on an analytical scale (Fig. 3). Having overcome this demanding separation problem in principle, upscaling to a semi-preparative scale has not been conducted yet. Observed yields and diastereoselectivities of performed indium-mediated allylation reactions are presented in Table 1.

\section{Conclusion}

We developed a straightforward method for the synthesis of 3-deoxy-2-uloses. Elongation of simple aldoses via an indium-mediated allylation reaction introduced a
2-C-methylidenepropan-1-ol motif. Depending on the initial substrate, epimer separation by column chromatography was facilitated via protecting group manipulations. In case of D-mannose, a benzyl protected allyl species was employed for the elongation to introduce UV-activity to the compounds, thus allowing for separation of the epimeric mixture using HPLC. Subsequent ozonolysis gave the respective 3-deoxy-2-ulose in good to excellent yields. By this method the stereochemical outcome can be controlled by the introduction of an appropriate protecting group in $\alpha$-position to the carbonyl function prior to the elongation, emphasizing the indium-mediated allylation reaction as a powerful tool for the synthesis of 3-deoxy-2-uloses.

\section{Experimental}

${ }^{1} \mathrm{H}$ and ${ }^{13} \mathrm{C}$ NMR spectra were recorded on a Bruker AV III 400, Bruker AV III 600, or Bruker AV III HD 700 respectively. Chemical shifts $(\delta)$ are reported in parts per million $(\mathrm{ppm})$ and spectra were calibrated using solvent signals of $\mathrm{CDCl}_{3}$ or $\mathrm{D}_{2} \mathrm{O}$. Signal multiplicity is indicated by one or more of the following: s (singlet); d (doublet); $t$ (triplet); q (quartet); m (multiplet); br (broad). NMR analysis was assisted by the spin simulation software DAISY (part of Bruker Top Spin software). Assignment of anomers was achieved by NOESY-NMR experiments. High resolution mass spectra (HRMS) were recorded on a Bruker maXis UHR-TOF spectrometer in ESI mode. Optical rotations were measured on a Schmidt-Haensch Digital Polarimeter Unipol

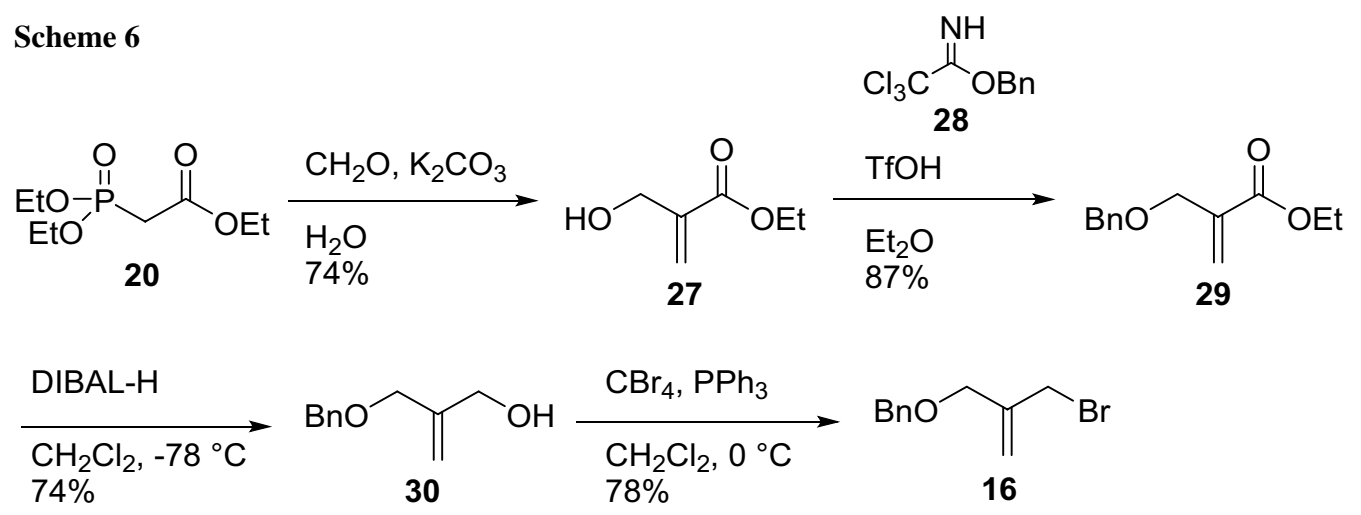

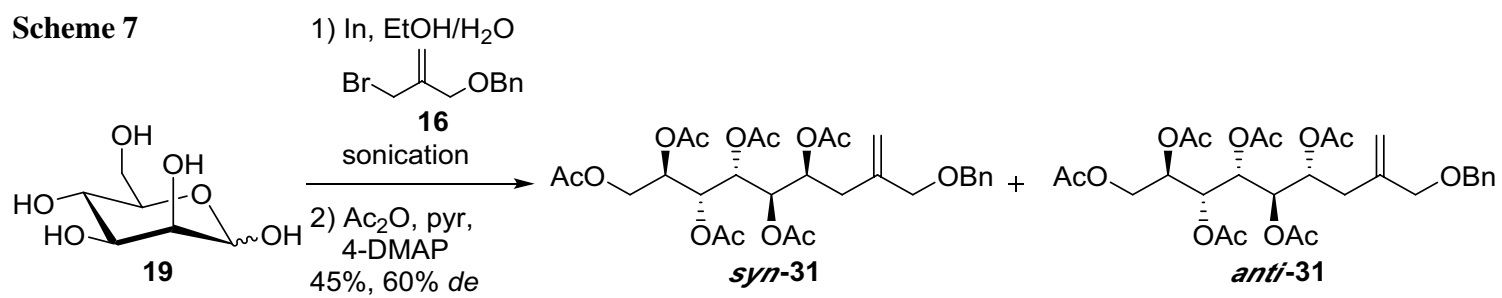




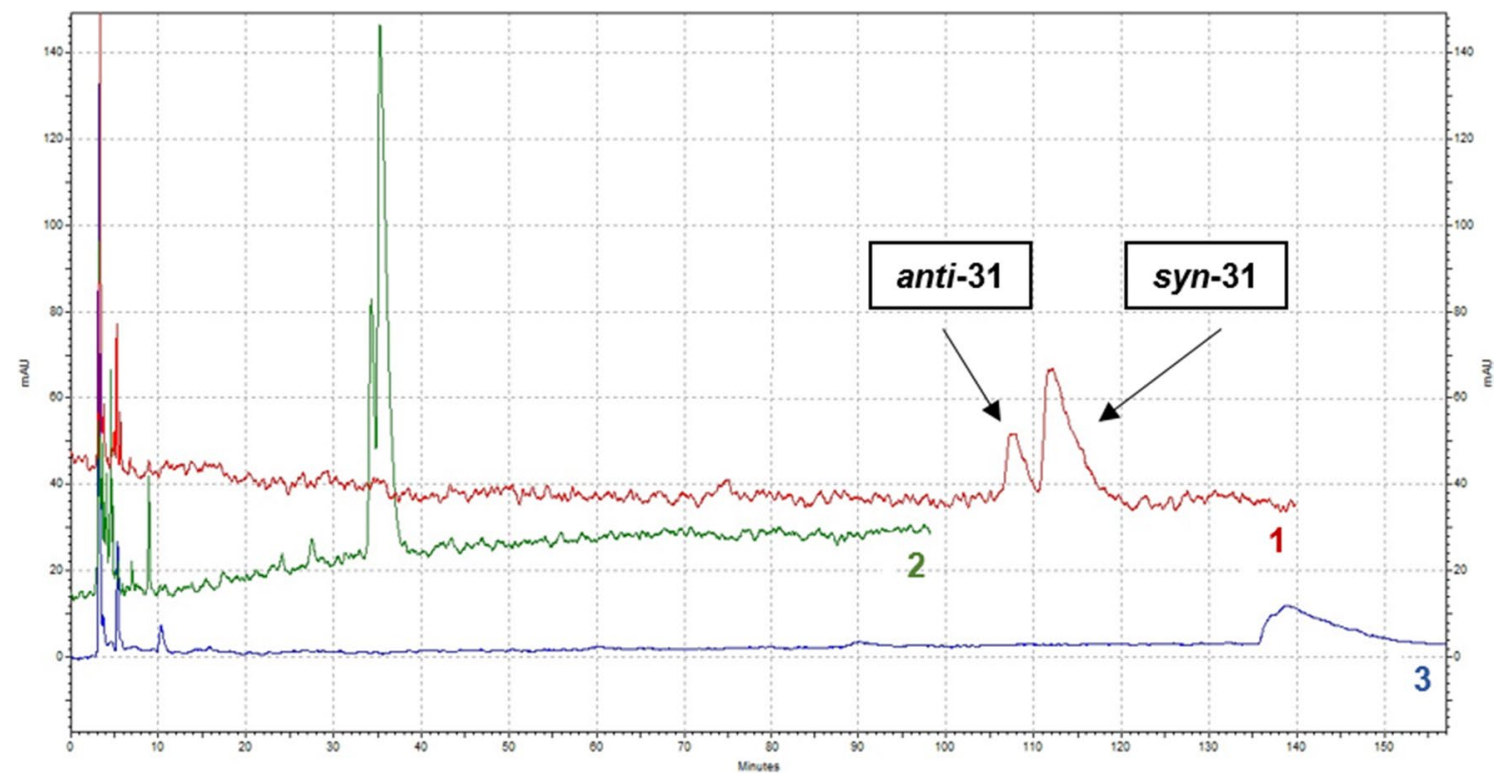

Fig. 3 Separation of epimeric mixture (syn-/anti-31) by HPLC: Macherey-Nagel NUCLEODUR ${ }^{\circledR} 100-5$ column, $15{ }^{\circ} \mathrm{C}$ column temperature, detection at $254 \mathrm{~nm}$, eluents: 1: heptane/ethyl acetate $=86 / 14,2$ : heptane/ethyl acetate $=80 / 20,3$ : heptane/ethyl acetate $=87 / 13$

Table 1 Yields and diastereoselectivities observed in the indiummediated allylation reactions

\begin{tabular}{|c|c|c|c|c|}
\hline Starting material & Allyl species & Product & $\begin{array}{l}\text { Com- } \\
\text { bined } \\
\text { yield/\% }\end{array}$ & Syn/anti \\
\hline $\begin{array}{l}\text { 2,3-O-isopropylidene- } \\
\text { D-glyceraldehyde } \\
\text { (17) }\end{array}$ & 14 & anti-21 & 75 & $1: 3$ \\
\hline D-arabinose (18) & 14 & syn-23 & $80^{\mathrm{a}}$ & $3: 1$ \\
\hline D-mannose (19) & 14 & syn- $25^{\mathrm{b}}$ & $60^{\mathrm{a}}$ & $5: 1$ \\
\hline D-mannose (19) & 16 & syn-31 ${ }^{b}$ & $45^{\mathrm{a}}$ & $4: 1$ \\
\hline
\end{tabular}

${ }^{\mathrm{a} C}$ Combined yields after allylation and subsequent per- $O$-acetylation

${ }^{\mathrm{b}}$ Referring to results obtained by us $[5-7,9,14,16]$ and others $[1-4$, $10-13,15]$, which confirm the influence of the strong chelating effect of a hydroxyl group in $\alpha$-position to the carbonyl function, we termed the major products $\mathbf{s y n - 2 5}$ and $\mathbf{s y n - 3 1}$, respectively

L 2000. Sonication was performed using a Bandelin Sonorex Digiplus DL $512 \mathrm{H}$ ultrasonic unit. Ozonolysis was executed operating an Anseros COM-AD-04 ozone generator. HPLC analyses were carried out on a Knauer system using Macherey-Nagel NUCLEODUR ${ }^{\circledR} 100-5$ and NUCLEODUR ${ }^{\circledR}$ $100-3 \mathrm{C}_{18}$ ec columns. Column chromatography was performed using Macherey-Nagel silica gel $60(0.04-0.063 \mathrm{~mm}$, 240-400 mesh). TLC monitoring was carried out on precoated Merck silica gel $60 \mathrm{~F}_{254}$ glass plates. Compounds were visualized by treatment with a $\mathrm{Mo}-\mathrm{Ce}\left(\mathrm{SO}_{4}\right)_{2}$ complex

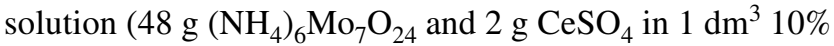
$\mathrm{H}_{2} \mathrm{SO}_{4}$ ) followed by heating. Filtrations over celite were performed with Celite ${ }^{\circledR}$ S by Sigma-Aldrich ${ }^{\circledR}$. Solvents were distilled, if necessary, prior to use. Reagents were purchased from commercial suppliers and were used without further purification.

Ethyl 2-(bromomethyl)acrylate A known procedure [25] was used for the synthesis of ethyl 2-(bromomethyl)acrylate. Starting from $33.63 \mathrm{~g}$ triethyl phosphonoacetate (20, $150.0 \mathrm{mmol}), 18.43 \mathrm{~g}$ of ethyl 2-(bromomethyl)acrylate $(95.46 \mathrm{mmol})$ with a yield of $64 \%$ over 2 steps was obtained. NMR signals correspond to published NMR data [25].

2-(Bromomethyl)prop-2-en-1-ol (14) A known procedure [26] was used for the synthesis of compound 14. Starting from $5.02 \mathrm{~g}$ ethyl 2-(bromomethyl)acrylate $(26.0 \mathrm{mmol})$, $2.40 \mathrm{~g}$ of compound $\mathbf{1 4}(15.9 \mathrm{mmol})$ with a yield of $61 \%$ was obtained. NMR signals correspond to published NMR data [26].

2,3-0-Isopropylidene-D-glyceraldehyde (17) Compound 17 was prepared according to a known isopropylidene protection procedure [27] and oxidative 1,2-diol cleavage protocol [28]. Starting from $25.10 \mathrm{~g}$ D-mannitol (137.8 mmol), $2.706 \mathrm{~g}$ of compound $\mathbf{1 7}(207.9 \mathrm{mmol})$ was obtained with a yield of $75 \%$ over 2 steps. NMR signals were found to correspond to NMR data in the literature [28].

2,3-Dideoxy-5,6-O-isopropylidene-2-C-methylidene-D-erythro-hexitol (anti-21, $\mathbf{C}_{\mathbf{1 0}} \mathbf{H}_{\mathbf{1 8}} \mathbf{O}_{\mathbf{4}}$ ) 2,3-O-Isopropylidene-D-glyceraldehyde $(\mathbf{1 7}, 108 \mathrm{mg}, 0.830 \mathrm{mmol})$ and $175 \mathrm{mg}$ 2-(bromomethyl)prop-2-en-1-ol (14, $1.16 \mathrm{mmol}$, 1.4 equiv.) were dissolved in $5 \mathrm{~cm}^{3}$ ethanol. Indium powder (133 mg, $1.16 \mathrm{mmol}, 1.4$ equiv.) was added and the 
suspension was sonicated at $45{ }^{\circ} \mathrm{C}$ for $18 \mathrm{~h}$. The reaction mixture was cooled to room temperature and the $\mathrm{pH}$ was adjusted to 7 with $1 \mathrm{M}$ aqueous sodium hydroxide solution. The suspension was filtered over a plug of celite which was subsequently rinsed with $20 \mathrm{~cm}^{3}$ ethanol. The filtrate was concentrated under reduced pressure and the epimeric mixture was purified by column chromatography with heptane/ethyl acetate $(1: 3 \mathrm{v} / \mathrm{v})$ as eluent to give $97 \mathrm{mg}$ compound anti-21 (0.48 mmol) as white solid and $29 \mathrm{mg}$ syn-21 $(0.143 \mathrm{mmol})$ as colorless oil $(75 \%, 54 \% \mathrm{de})$.

anti-21: ${ }^{1} \mathrm{H}$ NMR $\left(600.25 \mathrm{MHz}, \mathrm{CDCl}_{3}, 25{ }^{\circ} \mathrm{C}\right): \delta=5.118$ (s, 1H, H-1'a), 4.987 (s, 1H, H-1'b), 4.067 (m, 2H, H-1), 4.014 (m, 1H, H-6a), 4.964 (m, 1H, H-5), 3.907 (m, 1H, H-6b), 3.741 (m, 1H, H-4), 3.492 (s br, 1H, OH), 3.320 (s br, $1 \mathrm{H}, \mathrm{OH}), 2.435\left(\mathrm{~d},{ }^{2} J_{3 \mathrm{a}, 3 \mathrm{~b}}=14.3 \mathrm{~Hz}, 1 \mathrm{H}, \mathrm{H}-3 \mathrm{a}\right), 2.137$ (dd, $\left.{ }^{2} J_{3 \mathrm{~b}, 3 \mathrm{a}}=14.3 \mathrm{~Hz},{ }^{2} J_{3 \mathrm{~b}, 4}=9.2 \mathrm{~Hz}, 1 \mathrm{H}, \mathrm{H}-3 \mathrm{~b}\right), 1.401$ (s, $\left.3 \mathrm{H}, \mathrm{CH}_{3}\right), 1.333\left(\mathrm{~s}, 3 \mathrm{H}, \mathrm{CH}_{3}\right) \mathrm{ppm} ;{ }^{13} \mathrm{C} \mathrm{NMR}(150.93 \mathrm{MHz}$, $\left.\mathrm{CDCl}_{3}, 25{ }^{\circ} \mathrm{C}\right): \delta=145.21(\mathrm{C}-2), 114.89\left(\mathrm{C}-1^{\prime}\right), 109.33$ $\left(\underline{\mathrm{C}}\left(\mathrm{CH}_{3}\right)_{2}\right), 78.41(\mathrm{C}-5), 71.27(\mathrm{C}-4), 66.41(\mathrm{C}-1), 65.90$ (C-6), $37.93(\mathrm{C}-3), 26.69\left(\mathrm{CH}_{3}\right), 25.34\left(\mathrm{CH}_{3}\right)$ ppm; HRMS $\left(\mathrm{ESI}^{+}\right): \mathrm{m} / z=225.1102\left([\mathrm{M}+\mathrm{Na}]^{+}\right)$, calcd. for $\mathrm{C}_{10} \mathrm{H}_{18} \mathrm{NaO}_{4}{ }^{+}$ 225.1097; $[\alpha]_{D}^{20}=+0.5$ ( $c=6.5$, methanol); $R_{f}=0.5$ (heptane/ethyl acetate $=1: 3$ ).

syn-21: ${ }^{1} \mathrm{H}$ NMR $\left(600.25 \mathrm{MHz}, \mathrm{CDCl}_{3}, 25{ }^{\circ} \mathrm{C}\right): \delta=5.134$ (s, 1H, H-1'a), 4.972 (s, 1H, H-1'b), 4.156-4.097 (m, 2H, H-1), 4.089-4.003 (m, 2H, H-5, H-6a), 3.819-3.746 (m, 1H, H-6b), 3.745-3.655 (m, 1H, H-4), 3.492 (s br, 1H, OH), $3.320(\mathrm{~s} \mathrm{br}, 1 \mathrm{H}, \mathrm{OH}), 2.434\left(\mathrm{~d},{ }^{2} J_{3 \mathrm{a}, 3 \mathrm{~b}}=14.2 \mathrm{~Hz}, 1 \mathrm{H}, \mathrm{H}-3 \mathrm{a}\right)$, $2.137\left(\mathrm{dd},{ }^{2} J_{3 \mathrm{~b}, 3 \mathrm{a}}=14.2 \mathrm{~Hz},{ }^{3} J_{3 \mathrm{~b}, 4}=9.2 \mathrm{~Hz}, 1 \mathrm{H}, \mathrm{H}-3 \mathrm{~b}\right)$, $1.401\left(\mathrm{~s}, 3 \mathrm{H}, \mathrm{CH}_{3}\right), 1.333\left(\mathrm{~s}, 3 \mathrm{H}, \mathrm{CH}_{3}\right) \mathrm{ppm} ;{ }^{13} \mathrm{C} \mathrm{NMR}$ $\left(150.93 \mathrm{MHz}, \mathrm{CDCl}_{3}, 25^{\circ} \mathrm{C}\right): \delta=145.41(\mathrm{C}-2), 114.62$ $\left(\mathrm{C}-1^{\prime}\right), 109.77\left(\underline{\mathrm{C}}\left(\mathrm{CH}_{3}\right)_{2}\right), 78.69(\mathrm{C}-5), 71.90(\mathrm{C}-4), 66.58$ (C-1), 66.17 (C-6), 38.17 (C-3), $26.78\left(\mathrm{CH}_{3}\right), 25.42\left(\mathrm{CH}_{3}\right)$ ppm; HRMS $\left(\mathrm{ESI}^{+}\right): \mathrm{m} / z=225.1098\left([\mathrm{M}+\mathrm{Na}]^{+}\right)$, calcd. for $\mathrm{C}_{10} \mathrm{H}_{18} \mathrm{NaO}_{4}{ }^{+} 225.1097 ;[\alpha]_{D}^{20}=+0.8(c=2.6$, methanol); $R_{f}=0.4$ (heptane/ethyl acetate $=1: 3$ ).

\section{2,3-Dideoxy-2-C-methylidene-D-erythro-hexitol (22,} $\left.\mathbf{C}_{\mathbf{7}} \mathbf{H}_{14} \mathbf{O}_{\mathbf{4}}\right)$ Compound anti-21 (182 $\left.\mathrm{mg}, 0.900 \mathrm{mmol}\right)$ was dissolved in $5 \mathrm{~cm}^{3}$ water and $5 \mathrm{~cm}^{3}$ tetrahydrofuran and the $\mathrm{pH}$ was adjusted to 4 with $1 \mathrm{M}$ aqueous hydrochloride solution. After complete consumption of the starting material as monitored by TLC, the solution was neutralized using saturated aqueous sodium hydrogen carbonate solution. The mixture was concentrated and purified by column chromatography with dichloromethane/methanol $(4: 1 \mathrm{v} / \mathrm{v})$ as eluent to give $126 \mathrm{mg}$ compound $22(0.777 \mathrm{mmol}, 86 \%)$ as colorless oil. ${ }^{1} \mathrm{H}$ NMR $\left(600.25 \mathrm{MHz}, \mathrm{D}_{2} \mathrm{O}, 25^{\circ} \mathrm{C}\right): \delta=5.148$ (s, 1H, H-1'a), 5.035 (s, 1H, H-1'b), 4.122-4.033 (m, 2H, H-1), 3.844-3.736 (m, 2H, H-4, H-6a), 3.638-3.583 (m,
$2 \mathrm{H}, \mathrm{H}-5, \mathrm{H}-6 \mathrm{~b}$ ), $2.462\left(\mathrm{dd},{ }^{2} J_{3 \mathrm{a}, 3 \mathrm{~b}}=14.8 \mathrm{~Hz},{ }^{3} J_{3 \mathrm{a}, 4}=2.3 \mathrm{~Hz}\right.$, $1 \mathrm{H}, \mathrm{H}-3 \mathrm{a}), 2.154\left(\mathrm{dd},{ }^{2} J_{3 \mathrm{~b}, 3 \mathrm{a}}=14.8 \mathrm{~Hz},{ }^{3} J_{3 \mathrm{~b}, 4}=10.2 \mathrm{~Hz}\right.$, $1 \mathrm{H}, \mathrm{H}-3 \mathrm{a}) \mathrm{ppm} ;{ }^{13} \mathrm{C}$ NMR $\left(150.93 \mathrm{MHz}, \mathrm{D}_{2} \mathrm{O}, 25^{\circ} \mathrm{C}\right)$ : $\delta=144.72(\mathrm{C}-2), 112.63\left(\mathrm{C}-1^{\prime}\right), 74.60(\mathrm{C}-5), 69.92(\mathrm{C}-4)$, 64.28 (C-1), 62.42 (C-6), 35.79 (C-3) ppm; HRMS (ESI $\left.{ }^{+}\right)$: $m / z=185.0774\left([\mathrm{M}+\mathrm{Na}]^{+}\right)$, calcd. for $\mathrm{C}_{7} \mathrm{H}_{14} \mathrm{NaO}_{4}{ }^{+}$ $185.0784 ;[\alpha]_{D}^{20}=-7.8(c=0.3$, methanol $) ; R_{f}=0.5$ (dichloromethane/methanol $=4: 1$ ).

3-Deoxy-D-erythro-hexulose (5) Compound 22 (126 mg, $0.777 \mathrm{mmol}$ ) was dissolved in $6 \mathrm{~cm}^{3}$ methanol and $6 \mathrm{~cm}^{3}$ dichloromethane and the solution was cooled to $-78^{\circ} \mathrm{C}$. Ozone was led through the reaction mixture via a gas inlet tube until the solution's color turned yellow. The mixture was purged with air for $10 \mathrm{~min}$ and $171 \mathrm{~mm}^{3}$ of dimethyl sulfide ( $2.33 \mathrm{mmol}, 3$ equiv.) was added. The reaction solution was slowly warmed to $0{ }^{\circ} \mathrm{C}$ and the $\mathrm{pH}$ was adjusted to 7 with $1 \mathrm{M}$ aqueous sodium hydroxide solution. The cooling bath was removed and the mixture was stirred at ambient temperature for $6 \mathrm{~h}$. The mixture was concentrated and purified by column chromatography with dichloromethane/methanol $(4: 1 \mathrm{v} / \mathrm{v})$ as eluent to give $91 \mathrm{mg}$ compound $5(0.554 \mathrm{mmol}$, $71 \%)$ as colorless oil. ${ }^{1} \mathrm{H}$ NMR $\left(700.40 \mathrm{MHz}, \mathrm{D}_{2} \mathrm{O}\right.$, $25{ }^{\circ} \mathrm{C}$ ): $\beta$-pyranose: $\alpha$-furanose:keto isomer: $\beta$-furanose: $\alpha$ pyranose $=1.00: 0.40: 0.32: 0.30: 0.17 ; \delta(\beta$-pyranose $)=4.118$ (ddd, ${ }^{3} J_{4,3 \mathrm{a}}=12.5 \mathrm{~Hz},{ }^{3} J_{4,3 \mathrm{~b}}=5.0 \mathrm{~Hz},{ }^{3} J_{4,5}=3.3 \mathrm{~Hz}, 1 \mathrm{H}$, $\mathrm{H}-4), 4.026\left(\mathrm{dd},{ }^{2} J_{6 \mathrm{a}, 6 \mathrm{~b}}=12.8 \mathrm{~Hz},{ }^{3} J_{6 \mathrm{a}, 5}=1.4 \mathrm{~Hz}, 1 \mathrm{H}, \mathrm{H}-6 \mathrm{a}\right)$, $3.875\left(\mathrm{ddd},{ }^{3} J_{5,4}=3.3 \mathrm{~Hz},{ }^{3} J_{5,6 \mathrm{~b}}=2.2 \mathrm{~Hz},{ }^{3} J_{5,6 \mathrm{a}}=1.4 \mathrm{~Hz}\right.$, $1 \mathrm{H}, \mathrm{H}-5), 3.765\left(\mathrm{dd},{ }^{2} J_{6 \mathrm{~b}, 6 \mathrm{a}}=12.8 \mathrm{~Hz},{ }^{3} J_{6 \mathrm{~b}, 5}=2.2 \mathrm{~Hz}, 1 \mathrm{H}\right.$, $\mathrm{H}-6 \mathrm{~b}), 3.516\left(\mathrm{~d},{ }^{2} J_{1 \mathrm{a}, 1 \mathrm{~b}}=11.6 \mathrm{~Hz}, 1 \mathrm{H}, \mathrm{H}-1 \mathrm{a}\right), 3.510$ (d, $\left.{ }^{2} J_{1 \mathrm{~b}, 1 \mathrm{a}}=11.6 \mathrm{~Hz}, 1 \mathrm{H}, \mathrm{H}-1 \mathrm{~b}\right), 1.832\left(\mathrm{dd},{ }^{2} J_{3 \mathrm{a}, 3 \mathrm{~b}}=13.6 \mathrm{~Hz}\right.$, $\left.{ }^{3} J_{3 \mathrm{a}, 4}=12.5 \mathrm{~Hz}, 1 \mathrm{H}, \mathrm{H}-3 \mathrm{a}\right), 1.823\left(\mathrm{dd},{ }^{2} J_{3 \mathrm{~b}, 3 \mathrm{a}}=13.6 \mathrm{~Hz}\right.$, $\left.{ }^{3} J_{3 \mathrm{~b}, 4}=5.0 \mathrm{~Hz}, 1 \mathrm{H}, \mathrm{H}-3 \mathrm{~b}\right) \mathrm{ppm} ; \delta(\alpha$-furanose) $=4.277$ (ddd, $\left.{ }^{3} J_{4,3 \mathrm{a}}=7.4 \mathrm{~Hz},{ }^{3} J_{4,5}=4.5 \mathrm{~Hz},{ }^{3} J_{4,3 \mathrm{~b}}=4.1 \mathrm{~Hz}, 1 \mathrm{H}, \mathrm{H}-4\right), 4.172$ (ddd, ${ }^{3} J_{5,6 \mathrm{~b}}=5.6 \mathrm{~Hz},{ }^{3} J_{5,4}=4.5 \mathrm{~Hz},{ }^{3} J_{5,6 \mathrm{a}}=3.7 \mathrm{~Hz}, 1 \mathrm{H}, \mathrm{H}-5$ ), $3.725\left(\mathrm{dd},{ }^{2} J_{6 \mathrm{a}, 6 \mathrm{~b}}=12.3 \mathrm{~Hz},{ }^{3} J_{6 \mathrm{a}, 5}=3.7 \mathrm{~Hz}, 1 \mathrm{H}, \mathrm{H}-6 \mathrm{a}\right), 3.619$ $\left(\mathrm{dd},{ }^{2} J_{6 \mathrm{~b}, 6 \mathrm{a}}=12.3 \mathrm{~Hz},{ }^{3} J_{6 \mathrm{~b}, 5}=5.6 \mathrm{~Hz}, 1 \mathrm{H}, \mathrm{H}-6 \mathrm{~b}\right), 3.569(\mathrm{~d}$, $\left.{ }^{2} J_{1 \mathrm{a}, 1 \mathrm{~b}}=11.9 \mathrm{~Hz}, 1 \mathrm{H}, \mathrm{H}-1 \mathrm{a}\right), 3.562\left(\mathrm{~d},{ }^{2} J_{1 \mathrm{~b}, 1 \mathrm{a}}=11.9 \mathrm{~Hz}\right.$, $1 \mathrm{H}, \mathrm{H}-1 \mathrm{~b}), 2.446\left(\mathrm{dd},{ }^{2} J_{3 \mathrm{a}, 3 \mathrm{~b}}=14.2 \mathrm{~Hz},{ }^{3} J_{3 \mathrm{a}, 4}=7.4 \mathrm{~Hz}\right.$, $1 \mathrm{H}, \mathrm{H}-3 \mathrm{a}), 1.951\left(\mathrm{dd},{ }^{2} J_{3 \mathrm{~b}, 3 \mathrm{a}}=14.2 \mathrm{~Hz},{ }^{3} J_{3 \mathrm{~b}, 4}=4.1 \mathrm{~Hz}, 1 \mathrm{H}\right.$, $\mathrm{H}-3 \mathrm{~b})$ ppm; $\delta$ (keto isomer) $=4.433\left(\mathrm{~d},{ }^{2} J_{1 \mathrm{a}, 1 \mathrm{~b}}=19.0 \mathrm{~Hz}, 1 \mathrm{H}\right.$, $\mathrm{H}-1 \mathrm{a}), 4.370\left(\mathrm{~d},{ }^{2} J_{1 \mathrm{~b}, 1 \mathrm{a}}=19.0 \mathrm{~Hz}, 1 \mathrm{H}, \mathrm{H}-1 \mathrm{~b}\right), 4.112$ (ddd, $\left.{ }^{3} J_{4,3 \mathrm{~b}}=9.2 \mathrm{~Hz},{ }^{3} J_{4,5}=6.6 \mathrm{~Hz},{ }^{3} J_{4,3 \mathrm{a}}=3.5 \mathrm{~Hz}, 1 \mathrm{H}, \mathrm{H}-4\right)$, $3.750\left(\mathrm{dd},{ }^{2} J_{6 \mathrm{a}, 6 \mathrm{~b}}=11.8 \mathrm{~Hz},{ }^{3} J_{6 \mathrm{a}, 5}=3.3 \mathrm{~Hz}, 1 \mathrm{H}, \mathrm{H}-6 \mathrm{a}\right)$, $3.626\left(\mathrm{ddd},{ }^{3} J_{5,6 \mathrm{~b}}=7.0 \mathrm{~Hz},{ }^{3} J_{5,4}=6.6 \mathrm{~Hz},{ }^{3} J_{5,6 \mathrm{a}}=3.3 \mathrm{~Hz}\right.$, $1 \mathrm{H}, \mathrm{H}-5), 3.593\left(\mathrm{dd},{ }^{2} J_{6 \mathrm{~b}, 6 \mathrm{a}}=11.8 \mathrm{~Hz},{ }^{3} J_{6 \mathrm{~b}, 5}=7.0 \mathrm{~Hz}, 1 \mathrm{H}\right.$, $\mathrm{H}-6 \mathrm{~b}), 2.763\left(\mathrm{dd},{ }^{2} J_{3 \mathrm{a}, 3 \mathrm{~b}}=16.2 \mathrm{~Hz},{ }^{3} J_{3 \mathrm{a}, 4}=3.5 \mathrm{~Hz}, 1 \mathrm{H}\right.$, $\mathrm{H}-3 \mathrm{a}), 2.683\left(\mathrm{dd},{ }^{2} J_{3 \mathrm{~b}, 3 \mathrm{a}}=16.2 \mathrm{~Hz},{ }^{3} J_{3 \mathrm{~b}, 4}=9.2 \mathrm{~Hz}, 1 \mathrm{H}\right.$, $\mathrm{H}-3 \mathrm{~b}) \mathrm{ppm} ; \delta$ ( $\beta$-furanose $)=4.429\left(\mathrm{ddd},{ }^{3} J_{4,3 \mathrm{~b}}=7.2 \mathrm{~Hz}\right.$, $\left.{ }^{3} J_{4,3 \mathrm{a}}=7.1 \mathrm{~Hz},{ }^{3} J_{4,5}=5.5 \mathrm{~Hz}, 1 \mathrm{H}, \mathrm{H}-4\right), 3.950(\mathrm{ddd}$, $\left.{ }^{3} J_{5,6 \mathrm{~b}}=6.2 \mathrm{~Hz},{ }^{3} J_{5,4}=5.5 \mathrm{~Hz},{ }^{3} J_{5,6 \mathrm{a}}=3.9 \mathrm{~Hz}, 1 \mathrm{H}, \mathrm{H}-5\right)$, $3.770\left(\mathrm{dd},{ }^{2} J_{6 \mathrm{a}, 6 \mathrm{~b}}=12.1 \mathrm{~Hz},{ }^{3} J_{6 \mathrm{a}, 5}=3.9 \mathrm{~Hz}, 1 \mathrm{H}, \mathrm{H}-6 \mathrm{a}\right), 3.673$ $\left(\mathrm{dd},{ }^{2} J_{6 \mathrm{a}, 6 \mathrm{~b}}=12.1 \mathrm{~Hz},{ }^{3} J_{6 \mathrm{~b}, 5}=6.2 \mathrm{~Hz}, 1 \mathrm{H}, \mathrm{H}-6 \mathrm{~b}\right), 3.602(\mathrm{~s}$, 
$2 \mathrm{H}, \mathrm{H}-1), 2.290\left(\mathrm{dd},{ }^{2} J_{3 \mathrm{a}, 3 \mathrm{~b}}=13.6 \mathrm{~Hz},{ }^{3} J_{3 \mathrm{a}, 4}=7.1 \mathrm{~Hz}, 1 \mathrm{H}\right.$, $\mathrm{H}-3 \mathrm{a}), 2.171\left(\mathrm{dd},{ }^{2} J_{3 \mathrm{~b}, 3 \mathrm{a}}=13.6 \mathrm{~Hz},{ }^{3} J_{3 \mathrm{~b}, 4}=7.2 \mathrm{~Hz}, 1 \mathrm{H}\right.$, $\mathrm{H}-3 \mathrm{~b}) \mathrm{ppm} ; \delta(\alpha$-pyranose $)=4.159\left(\mathrm{dddd},{ }^{3} J_{4,3 \mathrm{a}}=5.1 \mathrm{~Hz}\right.$, $\left.{ }^{3} J_{4,3 \mathrm{~b}}=3.4 \mathrm{~Hz},{ }^{3} J_{4,5}=3.1 \mathrm{~Hz},{ }^{3} J_{4,6 \mathrm{~b}}=0.9 \mathrm{~Hz}, 1 \mathrm{H}, \mathrm{H}-4\right), 3.937$ $\left(\mathrm{dd},{ }^{2} J_{6 \mathrm{a}, 6 \mathrm{~b}}=11.5 \mathrm{~Hz},{ }^{3} J_{6 \mathrm{a}, 5}=9.6 \mathrm{~Hz}, 1 \mathrm{H}, \mathrm{H}-6 \mathrm{a}\right), 3.814$ (ddd, $\left.{ }^{3} J_{5,6 \mathrm{a}}=9.6 \mathrm{~Hz},{ }^{3} J_{5,6 \mathrm{~b}}=4.9 \mathrm{~Hz},{ }^{3} J_{5,4}=3.1 \mathrm{~Hz}, 1 \mathrm{H}, \mathrm{H}-5\right), 3.662$ (ddd, ${ }^{2} J_{6 \mathrm{~b}, 6 \mathrm{a}}=11.5 \mathrm{~Hz},{ }^{3} J_{6 \mathrm{~b}, 5}=4.9 \mathrm{~Hz},{ }^{3} J_{6 \mathrm{~b}, 4}=0.9 \mathrm{~Hz}, 1 \mathrm{H}$, H-6b), $3.500\left(\mathrm{~d},{ }^{2} J_{1 \mathrm{a}, 1 \mathrm{~b}}=11.8 \mathrm{~Hz}, 1 \mathrm{H}, \mathrm{H}-1 \mathrm{a}\right), 3.470$ (d, $\left.{ }^{2} J_{1 \mathrm{~b}, 1 \mathrm{a}}=11.8 \mathrm{~Hz}, 1 \mathrm{H}, \mathrm{H}-1 \mathrm{~b}\right), 1.995\left(\mathrm{dd},{ }^{2} J_{3 \mathrm{a}, 3 \mathrm{~b}}=14.5 \mathrm{~Hz}\right.$, $\left.{ }^{3} J_{3 \mathrm{a}, 4}=5.1 \mathrm{~Hz}, 1 \mathrm{H}, \mathrm{H}-3 \mathrm{a}\right), 1.940\left(\mathrm{dd},{ }^{2} J_{3 \mathrm{~b}, 3 \mathrm{a}}=14.5 \mathrm{~Hz}\right.$, $\left.{ }^{3} J_{3 \mathrm{~b}, 4}=3.4 \mathrm{~Hz}, 1 \mathrm{H}, \mathrm{H}-3 \mathrm{~b}\right) \mathrm{ppm} ;{ }^{13} \mathrm{C}$ NMR $(176.12 \mathrm{MHz}$, $\left.\mathrm{D}_{2} \mathrm{O}, 25^{\circ} \mathrm{C}\right): \delta(\beta$-pyranose $)=97.40(\mathrm{C}-2), 67.53(\mathrm{C}-1)$, 67.25 (C-5), 64.95 (C-4), 64.01 (C-6), 32.31 (C-3) ppm; $\delta(\alpha$-furanose $)=105.84(\mathrm{C}-2), 85.74(\mathrm{C}-5), 71.31(\mathrm{C}-4)$, 65.18 (C-1), 61.52 (C-6), 41.49 (C-3) ppm; $\delta$ (keto isomer $)=211.83(\mathrm{C}-2), 74.34(\mathrm{C}-5), 68.00(\mathrm{C}-1), 67.88(\mathrm{C}-4)$, $62.41(\mathrm{C}-6), 41.45(\mathrm{C}-3) \mathrm{ppm} ; \delta(\beta$-furanose $)=105.49$ (C-2), 86.26 (C-5), 70.98 (C-4), 65.43 (C-1), 62.53 (C-6), $41.52(\mathrm{C}-3) \mathrm{ppm} ; \delta(\alpha-$ pyranose $)=96.49(\mathrm{C}-2), 66.82(\mathrm{C}-4)$, 66.15 (C-5), 65.79 (C-1), 58.72 (C-6), 34.39 (C-3) ppm; ${ }^{1} \mathrm{H}$ NMR signals correspond to published NMR data [33]. HRMS $\left(\mathrm{ESI}^{+}\right): m / z=187.0579\left([\mathrm{M}+\mathrm{Na}]^{+}\right)$, calcd. For $\mathrm{C}_{6} \mathrm{H}_{12} \mathrm{NaO}_{5}{ }^{+} 187.0577 ;[\alpha]_{D}^{20}=-3.4(c=1.1$, methanol); $R_{f}=0.4$ (dichloromethane $/$ methanol $=4: 1$ ).

\section{1,4,5,6,7,8-Hexa-O-acetyl-2,3-dideoxy-2-C-methyl-} idene-D-gluco-octitol (syn-23, $\mathbf{C}_{\mathbf{2 1}} \mathbf{H}_{\mathbf{3 0}} \mathbf{O}_{\mathbf{1 2}}$ ) 2-(Bromomethyl)prop-2-en-1-ol (14, $616 \mathrm{mg}, 4.08 \mathrm{mmol}, 1.5$ equiv.) was dissolved in $16 \mathrm{~cm}^{3}$ ethanol and $4 \mathrm{~cm}^{3}$ water. Indium powder (468 mg, $4.08 \mathrm{mmol}, 1.5$ equiv.) and $408 \mathrm{mg}$ D-arabinose $(\mathbf{1 8}, 2.72 \mathrm{mmol})$ were added and the suspension was sonicated at $45^{\circ} \mathrm{C}$ for $6 \mathrm{~h}$. The reaction mixture was filtered over celite which was subsequently rinsed with $20 \mathrm{~cm}^{3}$ ethanol. The filtrate was evaporated yielding a colorless oil. The oil was taken up in $10 \mathrm{~cm}^{3}$ pyridine, treated with $10 \mathrm{~cm}^{3}$ acetic anhydride, and cooled to $0{ }^{\circ} \mathrm{C}$. 4-(Dimethylamino)pyridine (17 $\mathrm{mg}, 0.14 \mathrm{mmol}, 0.05$ equiv.) was added and the solution was stirred for $3 \mathrm{~h}$ while reaching room temperature. After no further conversion was observed via TLC analysis, the reaction mixture was diluted with toluene forming a homogenous pressure-maximum azeotrope with pyridine [34], thus facilitating concentration. The residue was taken up in 30 $\mathrm{cm}^{3}$ ethyl acetate and was washed with water $\left(3 \times 10 \mathrm{~cm}^{3}\right)$ and $10 \mathrm{~cm}^{3}$ brine. The organic layer was dried over magnesium sulfate, filtered and concentrated yielding a colorless oil. Separation of the resulting epimers was achieved via column chromatography using heptane/ethyl acetate $(1: 1 \mathrm{v} / \mathrm{v})$ as eluent to give $779 \mathrm{mg}$ compound $\mathbf{s y n - 2 3}(1.64 \mathrm{mmol})$ as colorless oil and $250 \mathrm{mg}$ compound anti-23 $(0.527 \mathrm{mmol})$ as colorless oil $(80 \%, 51 \% d e)$. syn-23: ${ }^{1} \mathrm{H}$ NMR $\left(600.25 \mathrm{MHz}, \mathrm{CDCl}_{3}, 25{ }^{\circ} \mathrm{C}\right): \delta=5.422$ $\left(\mathrm{dd},{ }^{3} J_{6,7}=7.3 \mathrm{~Hz},{ }^{3} J_{6,5}=3.9 \mathrm{~Hz}, 1 \mathrm{H}, 6-\mathrm{H}\right), 5.278(\mathrm{dd}$, $\left.{ }^{3} J_{5,4}=6.9 \mathrm{~Hz},{ }^{3} J_{5,6}=3.9 \mathrm{~Hz}, 1 \mathrm{H}, 5-\mathrm{H}\right), 5.186(\mathrm{ddd}$, $\left.{ }^{3} J_{4,3 \mathrm{~b}}=9.2 \mathrm{~Hz},{ }^{3} J_{4,5}=6.9 \mathrm{~Hz},{ }^{3} J_{4,3 \mathrm{a}}=3.6 \mathrm{~Hz}, 1 \mathrm{H}, 4-\mathrm{H}\right), 5.141$ $\left(\mathrm{d},{ }^{2} J_{1^{\prime} \mathrm{a}, 1^{\prime} \mathrm{b}}=0.9 \mathrm{~Hz}, 1 \mathrm{H}, 1^{\prime}-\mathrm{Ha}\right), 5.059\left(\mathrm{ddd},{ }^{3} J_{7,6}=7.3 \mathrm{~Hz}\right.$, $\left.{ }^{3} J_{7,8 \mathrm{~b}}=5.4 \mathrm{~Hz},{ }^{3} J_{7,8 \mathrm{a}}=3.1 \mathrm{~Hz}, 1 \mathrm{H}, 7-\mathrm{H}\right), 5.028(\mathrm{~d}$, $\left.{ }^{2} J_{1^{\prime} \mathrm{b}, 1^{\prime} \mathrm{a}}=0.9 \mathrm{~Hz}, 1 \mathrm{H}, 1^{\prime}-\mathrm{Hb}\right), 4.608\left(\mathrm{~d},{ }^{2} J_{1 \mathrm{a}, 1 \mathrm{~b}}=13.2 \mathrm{~Hz}\right.$, $1 \mathrm{H}, 1-\mathrm{Ha}), 4.443\left(\mathrm{~d},{ }^{2} J_{1 \mathrm{~b}, 1 \mathrm{a}}=13.2 \mathrm{~Hz}, 1 \mathrm{H}, 1-\mathrm{Hb}\right), 4.244$ $\left(\mathrm{dd},{ }^{2} J_{8 \mathrm{a}, 8 \mathrm{~b}}=12.5 \mathrm{~Hz},{ }^{3} J_{8 \mathrm{a}, 7}=3.1 \mathrm{~Hz}, 1 \mathrm{H}, 8-\mathrm{Ha}\right), 4.117$ $\left(\mathrm{dd},{ }^{2} J_{8 \mathrm{~b}, 8 \mathrm{a}}=12.5 \mathrm{~Hz},{ }^{3} J_{8 \mathrm{~b}, 7}=5.4 \mathrm{~Hz}, 1 \mathrm{H}, 8-\mathrm{Hb}\right), 2.459$ $\left(\mathrm{dd},{ }^{2} J_{3 \mathrm{a}, 3 \mathrm{~b}}=14.3 \mathrm{~Hz},{ }^{3} J_{3 \mathrm{a}, 4}=3.6 \mathrm{~Hz}, 1 \mathrm{H}, 3-\mathrm{Ha}\right), 2.300(\mathrm{dd}$, $\left.{ }^{2} J_{3 \mathrm{~b}, 3 \mathrm{a}}=14.3 \mathrm{~Hz},{ }^{3} J_{3 \mathrm{~b}, 4}=9.2 \mathrm{~Hz}, 1 \mathrm{H}, 3-\mathrm{Hb}\right), 2.1304(\mathrm{~s}, 3 \mathrm{H}$, OAc), 2.0856 (s, 3H, OAc), 2.0620 (s, 3H, OAc), 2.0582 (s, 3H, OAc), 2.0491 (s, 3H, OAc), 2.0153 (s, 3H, OAc) ppm; ${ }^{13} \mathrm{C}$ NMR $\left(150.93 \mathrm{MHz}, \mathrm{CDCl}_{3}, 25{ }^{\circ} \mathrm{C}\right): \delta=170.58$ $\left((\underline{\mathrm{C}}=\mathrm{O})-\mathrm{CH}_{3}\right), 170.48\left((\underline{\mathrm{C}}=\mathrm{O})-\mathrm{CH}_{3}\right), 169.96((\underline{\mathrm{C}}=\mathrm{O})-$ $\left.\mathrm{CH}_{3}\right), 169.90\left((\underline{\mathrm{C}}=\mathrm{O})-\mathrm{CH}_{3}\right), 169.80\left((\underline{\mathrm{C}}=\mathrm{O})-\mathrm{CH}_{3}\right), 169.79$ $\left((\mathrm{C}=\mathrm{O})-\mathrm{CH}_{3}\right), 138.69(2-\mathrm{C}), 117.53\left(1^{\prime}-\mathrm{C}\right), 70.72(5-\mathrm{C})$, 69.24 (4-C), 68.63 (7-C), 68.59 (6-C), 66.22 (1-C), 61.49 (8-C), 34.73 (3-C), $20.86\left((\mathrm{C}=\mathrm{O})-\mathrm{CH}_{3}\right), 20.75((\mathrm{C}=\mathrm{O})-$ $\left.\mathrm{C}_{3}\right), 20.68\left((\mathrm{C}=\mathrm{O})-\underline{\mathrm{CH}}_{3}\right), 20.66\left((\mathrm{C}=\mathrm{O})-\underline{\mathrm{CH}}_{3}\right), 20.60$ $\left((\mathrm{C}=\mathrm{O})-\mathrm{CH}_{3}\right), 20.52\left((\mathrm{C}=\mathrm{O})-\mathrm{CH}_{3}\right) \mathrm{ppm}$; HRMS $\left(\mathrm{ESI}^{+}\right)$: $m / z=497.1634\left([\mathrm{M}+\mathrm{Na}]^{+}\right)$, calcd. for $\mathrm{C}_{21} \mathrm{H}_{30} \mathrm{O}_{12} \mathrm{Na}^{+}$ 497.1635; $[\alpha]_{D}^{20}=+0.3\left(c=1.0\right.$, dichloromethane); $R_{f}=0.4$ (heptane/ethyl acetate $=1: 1$ ).

anti-23: ${ }^{1} \mathrm{H}$ NMR $\left(400.27 \mathrm{MHz}, \mathrm{CDCl}_{3}, 25{ }^{\circ} \mathrm{C}\right)$ : $\delta=5.431\left(\mathrm{dd},{ }^{3} J_{6,7}=8.7 \mathrm{~Hz},{ }^{3} J_{6,5}=2.7 \mathrm{~Hz}, 1 \mathrm{H}, 6-\mathrm{H}\right)$, $5.272\left(\mathrm{dd},{ }^{3} J_{5,4}=7.5 \mathrm{~Hz},{ }^{3} J_{5,6}=2.7 \mathrm{~Hz}, 1 \mathrm{H}, 5-\mathrm{H}\right), 5.134$ $\left(\mathrm{ddd},{ }^{3} J_{4,3 \mathrm{a}}=8.2 \mathrm{~Hz},{ }^{3} J_{4,5}=7.5 \mathrm{~Hz},{ }^{3} J_{4,3 \mathrm{~b}}=4.5 \mathrm{~Hz}, 1 \mathrm{H}\right.$, 4-H), $5.093\left(\mathrm{~d},{ }^{2} J_{1^{\prime} \mathrm{a}, 1^{\prime} \mathrm{b}}=0.9 \mathrm{~Hz}, 1 \mathrm{H}, 1^{\prime}-\mathrm{Ha}\right), 5.068(\mathrm{ddd}$, $\left.{ }^{3} J_{7,6}=8.7 \mathrm{~Hz},{ }^{3} J_{7,8 \mathrm{~b}}=5.5 \mathrm{~Hz},{ }^{3} J_{7,8 \mathrm{a}}=2.8 \mathrm{~Hz}, 1 \mathrm{H}, 7-\mathrm{H}\right), 4.983$ $\left(\mathrm{d},{ }^{2} J_{1^{\prime} \mathrm{b}, 1^{\prime} \mathrm{a}}=0.9 \mathrm{~Hz}, 1 \mathrm{H}, 1^{\prime}-\mathrm{Hb}\right), 4.560\left(\mathrm{~d},{ }^{2} J_{1 \mathrm{a}, 1 \mathrm{~b}}=13.2 \mathrm{~Hz}\right.$, $1 \mathrm{H}, 1-\mathrm{Ha}), 4.515\left(\mathrm{~d},{ }^{2} J_{1 \mathrm{~b}, 1 \mathrm{a}}=13.2 \mathrm{~Hz}, 1 \mathrm{H}, 1-\mathrm{Hb}\right), 4.208$ $\left(\mathrm{dd},{ }^{2} J_{8 \mathrm{a}, 8 \mathrm{~b}}=12.5 \mathrm{~Hz},{ }^{3} J_{8 \mathrm{a}, 7}=2.8 \mathrm{~Hz}, 1 \mathrm{H}, 8-\mathrm{Ha}\right), 4.068$ $\left(\mathrm{dd},{ }^{2} J_{8 \mathrm{~b}, 8 \mathrm{a}}=12.5 \mathrm{~Hz},{ }^{3} J_{8 \mathrm{~b}, 7}=5.5 \mathrm{~Hz}, 1 \mathrm{H}, 8-\mathrm{Hb}\right), 2.333$ $\left(\mathrm{dd},{ }^{2} J_{3 \mathrm{a}, 3 \mathrm{~b}}=14.3 \mathrm{~Hz},{ }^{3} J_{3 \mathrm{a}, 4}=8.2 \mathrm{~Hz}, 1 \mathrm{H}, 3-\mathrm{Ha}\right), 2.306(\mathrm{dd}$, $\left.{ }^{2} J_{3 \mathrm{~b}, 3 \mathrm{a}}=14.3 \mathrm{~Hz},{ }^{3} J_{3 \mathrm{~b}, 4}=4.5 \mathrm{~Hz}, 1 \mathrm{H}, 3-\mathrm{Hb}\right), 2.085(\mathrm{~s}, 3 \mathrm{H}$, OAc), 2.077 (s, 3H, OAc), 2.073 (s, 3H, OAc), 2.053 (s, 3H, OAc), 2.039 (s, 3H, OAc), 1.988 (s, $3 \mathrm{H}, \mathrm{OAc}) \mathrm{ppm} ;{ }^{13} \mathrm{C}$ NMR $\left(100.66 \mathrm{MHz}, \mathrm{CDCl}_{3}, 25^{\circ} \mathrm{C}\right): \delta=170.58((\underline{\mathrm{C}}=\mathrm{O})-$ $\left.\mathrm{CH}_{3}\right), 170.56\left((\underline{\mathrm{C}}=\mathrm{O})-\mathrm{CH}_{3}\right), 170.06\left((\underline{\mathrm{C}}=\mathrm{O})-\mathrm{CH}_{3}\right), 169.90$ $\left((\underline{\mathrm{C}}=\mathrm{O})-\mathrm{CH}_{3}\right), 169.87\left((\underline{\mathrm{C}}=\mathrm{O})-\mathrm{CH}_{3}\right), 169.77\left((\underline{\mathrm{C}}=\mathrm{O})-\mathrm{CH}_{3}\right)$, 139.00 (2-C), 116.68 (1'-C), 70.57 (5-C), 68.12 (7-C), 68.04 (6-C), 67.56(4-C), 66.22 (1-C), 61.89 (8-C), 35.38 (3-C), 20.55-20.89 (6×(C=O)- $\left.\mathrm{CH}_{3}\right)$ ppm; HRMS $\left(\mathrm{ESI}^{+}\right)$: $m / z=497.1633\left([\mathrm{M}+\mathrm{Na}]^{+}\right)$, calcd. for $\mathrm{C}_{21} \mathrm{H}_{30} \mathrm{O}_{12} \mathrm{Na}^{+}$ 497.1629; $R_{f}=0.4$ (heptane/ethyl acetate $=1: 1$ ).

2,3-Dideoxy-2-C-methylidene-D-gluco-octitol (24, $\mathrm{C}_{\mathbf{9}} \mathrm{H}_{\mathbf{1 8}} \mathbf{O}_{\mathbf{6}}$ ) Compound syn-23 (133 mg, $0.280 \mathrm{mmol}$ ) was dissolved in $10 \mathrm{~cm}^{3}$ methanol and the solution was treated with $2 \mathrm{mg}$ sodium methoxide ( $0.037 \mathrm{mmol}, 0.13$ equiv.). 
The mixture was stirred for $3 \mathrm{~h}$ until no further conversion could be observed via TLC analysis. The solution was neutralized using DOWEX W50 ion exchange resin $\left(\mathrm{H}^{+}\right.$form) which was subsequently filtered. The filtrate was concentrated to give $62 \mathrm{mg}$ compound $\mathbf{2 4}(0.28 \mathrm{mmol},>99 \%)$ as a white solid. Single crystals were grown by recrystallization in water/acetone [29]. ${ }^{1} \mathrm{H}$ NMR $\left(600.25 \mathrm{MHz}, \mathrm{D}_{2} \mathrm{O}\right.$, $\left.25^{\circ} \mathrm{C}\right): \delta=5.159\left(\mathrm{~d},{ }^{2} J_{1^{\prime} \mathrm{a}, 1^{\circ} \mathrm{b}}=1.2 \mathrm{~Hz}, 1 \mathrm{H}, 1^{\prime}-\mathrm{Ha}\right), 5.046(\mathrm{~d}$, $\left.{ }^{2} J_{1^{\prime} \mathrm{b}, 1^{\prime} \mathrm{a}}=1.2 \mathrm{~Hz}, 1 \mathrm{H}, 1^{\prime}-\mathrm{Hb}\right), 4.098\left(\mathrm{~d},{ }^{2} J_{1 \mathrm{a}, 1 \mathrm{~b}}=14.5 \mathrm{~Hz}, 1 \mathrm{H}\right.$, $1-\mathrm{Ha}), 4.090\left(\mathrm{~d},{ }^{2} J_{1 \mathrm{~b}, 1 \mathrm{a}}=14.5 \mathrm{~Hz}, 1 \mathrm{H}, 1-\mathrm{Hb}\right), 3.941$ (ddd, $\left.{ }^{3} J_{4,3 \mathrm{~b}}=9.5 \mathrm{~Hz},{ }^{3} J_{4,5}=5.7 \mathrm{~Hz},{ }^{3} J_{4,3 \mathrm{a}}=3.9 \mathrm{~Hz}, 1 \mathrm{H}, 4-\mathrm{H}\right)$, $3.823\left(\mathrm{dd},{ }^{2} J_{8 \mathrm{a}, 8 \mathrm{~b}}=11.9 \mathrm{~Hz},{ }^{3} J_{8 \mathrm{a}, 7}=3.0 \mathrm{~Hz}, 1 \mathrm{H}, 8-\mathrm{Ha}\right)$, $3.772\left(\mathrm{ddd},{ }^{3} J_{7,6}=8.2 \mathrm{~Hz},{ }^{3} J_{7,8 \mathrm{~b}}=6.3 \mathrm{~Hz},{ }^{3} J_{7,8 \mathrm{a}}=3.0 \mathrm{~Hz}\right.$, $1 \mathrm{H}, 7-\mathrm{H}), 3.711\left(\mathrm{dd},{ }^{3} J_{5,4}=5.7 \mathrm{~Hz},{ }^{3} J_{5,6}=2.3 \mathrm{~Hz}, 1 \mathrm{H}\right.$, $5-\mathrm{H}), 3.689\left(\mathrm{dd},{ }^{3} J_{6,7}=8.2 \mathrm{~Hz},{ }^{3} J_{6,5}=2.3 \mathrm{~Hz}, 1 \mathrm{H}, 6-\mathrm{H}\right)$, $3.643\left(\mathrm{dd},{ }^{2} J_{8 \mathrm{~b}, 8 \mathrm{a}}=11.9 \mathrm{~Hz},{ }^{3} J_{8 \mathrm{~b}, 7}=6.3 \mathrm{~Hz}, 1 \mathrm{H}, 8-\mathrm{Hb}\right)$, $2.419\left(\mathrm{dd},{ }^{2} J_{3 \mathrm{a}, 3 \mathrm{~b}}=14.5 \mathrm{~Hz},{ }^{3} J_{3 \mathrm{a}, 4}=3.9 \mathrm{~Hz}, 1 \mathrm{H}, 3-\mathrm{Ha}\right)$, $2.216\left(\mathrm{dd},{ }^{2} J_{3 \mathrm{~b}, 3 \mathrm{a}}=14.5 \mathrm{~Hz},{ }^{3} J_{3 \mathrm{~b}, 4}=9.5 \mathrm{~Hz}, 1 \mathrm{H}, 3-\mathrm{Hb}\right) \mathrm{ppm}$; ${ }^{13} \mathrm{C}$ NMR $\left(150.93 \mathrm{MHz}, \mathrm{D}_{2} \mathrm{O}, 25{ }^{\circ} \mathrm{C}\right): \delta=144.47(2-\mathrm{C})$, 112.94 (1'-C), 71.96 (5-C), 71.32 (6-C), 71.05 (7-C), 70.59 (4-C), 64.25 (1-C), 62.78 (8-C), 36.29 (3-C) ppm; HRMS $\left(\mathrm{ESI}^{+}\right): \mathrm{m} / z=245.0998\left([\mathrm{M}+\mathrm{Na}]^{+}\right)$, calcd. for $\mathrm{C}_{9} \mathrm{H}_{18} \mathrm{O}_{6} \mathrm{Na}^{+}$ 245.0996; m.p.: $124-126^{\circ} \mathrm{C} ;[\alpha]_{D}^{20}=-0.1$ (c=1.0, water); $R_{f}=0.7$ (1-butanol/acetone $/$ water $\left.=4: 4: 2\right)$.

CCDC 1899885 contains the supplementary crystallographic data for compound 24. These data can be obtained from The Cambridge Crystallographic Data Centre, www. ccdc.cam.ac.uk/data_request/cif.

\section{3-Deoxy-D-gluco-octulose $\left(15, \mathrm{C}_{8} \mathrm{H}_{16} \mathrm{O}_{7}\right)$ Compound}

$24(51 \mathrm{mg}, 0.23 \mathrm{mmol}$ ) was dissolved in $10 \mathrm{~cm} 3$ methanol and $10 \mathrm{~cm} 3$ dichloromethane and the solution was cooled to $-78{ }^{\circ} \mathrm{C}$. Ozone was led through the reaction mixture via a gas inlet tube until the solution's color turned blue. The mixture was purged with air for $15 \mathrm{~min}$, treated with $121 \mathrm{mg}$ triphenylphosphine $(0.46 \mathrm{mmol}, 2.0$ equiv. $)$ and stirred overnight at room temperature. The solvents were removed under reduced pressure and the residue was taken up in $20 \mathrm{~cm}^{3}$ dichloromethane and $20 \mathrm{~cm}^{3}$ water. The aqueous phase was washed with dichloromethane $(3 \times 10$ $\mathrm{cm}^{3}$ ). The aqueous layer was concentrated and the resulting oil was purified by column chromatography using dichloromethane/methanol $(4: 1 \mathrm{v} / \mathrm{v})$ as eluent to give $40 \mathrm{mg}$ compound $15(0.18 \mathrm{mmol}, 78 \%)$ as a colorless oil. ${ }^{1} \mathrm{H}$ NMR (600.25 MHz, $\left.\mathrm{D}_{2} \mathrm{O}, 25{ }^{\circ} \mathrm{C}\right)$ : $\beta$-pyranose:keto isomer: $\alpha$-furanose: $\beta$-furanose: $=1.00: 0.19: 0.18: 0.09 ; \delta$ $(\beta$-pyranose $)=4.120\left(\mathrm{dd},{ }^{3} J_{6,7}=9.0 \mathrm{~Hz},{ }^{3} J_{6,5}=1.4 \mathrm{~Hz}\right.$, $1 \mathrm{H}, 6-\mathrm{H}), 4.111\left(\mathrm{ddd},{ }^{3} J_{4,3 \mathrm{a}}=3.7 \mathrm{~Hz},{ }^{3} J_{4,5}=3.6 \mathrm{~Hz}\right.$, $\left.{ }^{3} J_{4,3 \mathrm{~b}}=2.8 \mathrm{~Hz}, 1 \mathrm{H}, 4-\mathrm{H}\right), 3.862\left(\mathrm{ddd},{ }^{3} J_{5,4}=3.6 \mathrm{~Hz}\right.$, $\left.{ }^{3} J_{5,6}=1.4 \mathrm{~Hz},{ }^{4} J_{5,3 \mathrm{~b}}=1.0 \mathrm{~Hz}, 1 \mathrm{H}, 5-\mathrm{H}\right), 3.855$ (ddd, $\left.{ }^{3} J_{7,6}=9.0 \mathrm{~Hz},{ }^{3} J_{7,8 \mathrm{~b}}=6.0 \mathrm{~Hz},{ }^{3} J_{7,8 \mathrm{a}}=2.9 \mathrm{~Hz}, 1 \mathrm{H}, 7-\mathrm{H}\right), 3.835$ $\left(\mathrm{dd},{ }^{2} J_{8 \mathrm{a}, 8 \mathrm{~b}}=12.0 \mathrm{~Hz},{ }^{3} J_{8 \mathrm{a}, 7}=2.9 \mathrm{~Hz}, 1 \mathrm{H}, 8-\mathrm{Ha}\right), 3.664$ (dd, $\left.{ }^{2} J_{8 \mathrm{~b}, 8 \mathrm{a}}=12.0 \mathrm{~Hz},{ }^{3} J_{8 \mathrm{~b}, 7}=6.0 \mathrm{~Hz}, 1 \mathrm{H}, 8-\mathrm{Hb}\right), 3.456(\mathrm{~s}, 2 \mathrm{H}$, 1-Ha, 1-Hb), $2.024\left(\mathrm{dd},{ }^{2} J_{3 \mathrm{a}, 3 \mathrm{~b}}=14.9 \mathrm{~Hz},{ }^{3} J_{3 \mathrm{a}, 4}=3.7 \mathrm{~Hz}\right.$, $1 \mathrm{H}, 3-\mathrm{Ha}), 1.782\left(\mathrm{ddd},{ }^{2} J_{3 \mathrm{~b}, 3 \mathrm{a}}=14.9 \mathrm{~Hz},{ }^{3} J_{3 \mathrm{~b}, 4}=2.8 \mathrm{~Hz}\right.$, ${ }^{4} J_{3 \mathrm{~b}, 5}=1.0 \mathrm{~Hz}, 1 \mathrm{H}, 3-\mathrm{Hb}$ ) ppm; $\delta$ (keto isomer) $=4.435$ $\left(\mathrm{dd},{ }^{2} J_{1 \mathrm{a}, 1 \mathrm{~b}}=19.2 \mathrm{~Hz},{ }^{4} J_{1 \mathrm{a}, 3 \mathrm{~b}}=0.6 \mathrm{~Hz}, 1 \mathrm{H}, 1-\mathrm{Ha}\right), 4.380(\mathrm{dd}$, $\left.{ }^{2} J_{1 \mathrm{~b}, 1 \mathrm{a}}=19.2 \mathrm{~Hz},{ }^{4} J_{1 \mathrm{~b}, 3 \mathrm{~b}}=0.6 \mathrm{~Hz}, 1 \mathrm{H}, 1-\mathrm{Hb}\right), 4.257$ (ddd, $\left.{ }^{3} J_{4,3 \mathrm{a}}=9.4 \mathrm{~Hz},{ }^{3} J_{4,5}=5.7 \mathrm{~Hz},{ }^{3} J_{4,3 \mathrm{~b}}=3.5 \mathrm{~Hz}, 1 \mathrm{H}, 4-\mathrm{H}\right)$, $3.827\left(\mathrm{dd},{ }^{2} J_{8 \mathrm{a}, 8 \mathrm{~b}}=12.0 \mathrm{~Hz},{ }^{3} J_{8 \mathrm{a}, 7}=3.0 \mathrm{~Hz}, 1 \mathrm{H}, 8 \mathrm{a}-\mathrm{H}\right)$, $3.773\left(\mathrm{ddd},{ }^{3} J_{7,6}=8.2 \mathrm{~Hz},{ }^{3} J_{7,8 \mathrm{~b}}=6.3 \mathrm{~Hz},{ }^{3} J_{7,8 \mathrm{a}}=3.0 \mathrm{~Hz}\right.$, $1 \mathrm{H}, 7-\mathrm{H}), 3.740\left(\mathrm{dd},{ }^{3} J_{5,4}=5.7 \mathrm{~Hz},{ }^{3} J_{5,6}=2.4 \mathrm{~Hz}, 1 \mathrm{H}, 5-\mathrm{H}\right)$, $3.655\left(\mathrm{dd},{ }^{2} J_{8 \mathrm{~b}, 8 \mathrm{a}}=12.0 \mathrm{~Hz},{ }^{3} J_{8 \mathrm{~b}, 7}=6.3 \mathrm{~Hz}, 1 \mathrm{H}, 8-\mathrm{Hb}\right)$, $3.627\left(\mathrm{dd},{ }^{3} J_{6,7}=8.2 \mathrm{~Hz},{ }^{3} J_{6,5}=2.4 \mathrm{~Hz}, 1 \mathrm{H}, 6-\mathrm{H}\right), 2.750$ $\left(\mathrm{dd},{ }^{2} J_{3 \mathrm{a}, 3 \mathrm{~b}}=16.3 \mathrm{~Hz},{ }^{3} J_{3 \mathrm{a}, 4}=9.4 \mathrm{~Hz}, 1 \mathrm{H}, 3-\mathrm{Ha}\right), 2.713$ (dddd, ${ }^{2} J_{3 \mathrm{~b}, 3 \mathrm{a}}=16.3 \mathrm{~Hz},{ }^{3} J_{3 \mathrm{~b}, 4}=3.5 \mathrm{~Hz},{ }^{4} J_{3 \mathrm{~b}, 1 \mathrm{a}}=0.6 \mathrm{~Hz}$, $\left.{ }^{4} J_{3 \mathrm{~b}, 1 \mathrm{~b}}=0.6 \mathrm{~Hz}, 1 \mathrm{H}, 3-\mathrm{Hb}\right) \mathrm{ppm} ; \delta(\alpha$-furanose $)=4.614$ (ddd, ${ }^{3} J_{4,3 \mathrm{a}}=6.3 \mathrm{~Hz},{ }^{3} J_{4,5}=4.4 \mathrm{~Hz},{ }^{3} J_{4,3 \mathrm{~b}}=3.3 \mathrm{~Hz}, 1 \mathrm{H}$, $4-\mathrm{H}), 4.294\left(\mathrm{dd},{ }^{3} J_{5,6}=5.0 \mathrm{~Hz},{ }^{3} J_{5,4}=4.4 \mathrm{~Hz}, 1 \mathrm{H}, 5-\mathrm{H}\right)$, $3.921\left(\mathrm{dd},{ }^{3} J_{6,7}=6.8 \mathrm{~Hz},{ }^{3} J_{6,5}=5.0 \mathrm{~Hz}, 1 \mathrm{H}, 6-\mathrm{H}\right), 3.814$ $\left(\mathrm{dd},{ }^{2} J_{8 \mathrm{a}, 8 \mathrm{~b}}=12.0 \mathrm{~Hz},{ }^{3} J_{8 \mathrm{a}, 7}=3.3 \mathrm{~Hz}, 1 \mathrm{H}, 8-\mathrm{Ha}\right), 3.785$ $\left(\mathrm{ddd},{ }^{3} J_{7,8 \mathrm{~b}}=6.9 \mathrm{~Hz},{ }^{3} J_{7,6}=6.8 \mathrm{~Hz},{ }^{3} J_{7,8 \mathrm{a}}=3.3 \mathrm{~Hz}, 1 \mathrm{H}\right.$, $7-\mathrm{H}), 3.678\left(\mathrm{dd},{ }^{2} J_{8 \mathrm{~b}, 8 \mathrm{a}}=12.0 \mathrm{~Hz},{ }^{3} J_{8 \mathrm{~b}, 7}=6.9 \mathrm{~Hz}, 1 \mathrm{H}, 8-\mathrm{Hb}\right)$, $3.653(\mathrm{~s}, 2 \mathrm{H}, 1-\mathrm{Ha}, 1-\mathrm{Hb}), 2.305\left(\mathrm{dd},{ }^{2} J_{3 \mathrm{a}, 3 \mathrm{~b}}=14.5 \mathrm{~Hz}\right.$, $\left.{ }^{3} J_{3 \mathrm{a}, 4}=6.3 \mathrm{~Hz}, 1 \mathrm{H}, 3-\mathrm{Ha}\right), 2.241\left(\mathrm{dd},{ }^{2} J_{3 \mathrm{~b}, 3 \mathrm{a}}=14.5 \mathrm{~Hz}\right.$, $\left.{ }^{3} J_{3 \mathrm{~b}, 4}=3.3 \mathrm{~Hz}, 1 \mathrm{H}, 3-\mathrm{Hb}\right) \mathrm{ppm} ; \delta(\beta$-furanose $)=4.538$ (ddd, ${ }^{3} J_{4,3 \mathrm{a}}=5.6 \mathrm{~Hz},{ }^{3} J_{4,5}=4.1 \mathrm{~Hz},{ }^{3} J_{4,3 \mathrm{~b}}=1.9 \mathrm{~Hz}, 1 \mathrm{H}$, $4-\mathrm{H}), 4.122\left(\mathrm{dd},{ }^{3} J_{5,6}=5.3 \mathrm{~Hz},{ }^{3} J_{5,4}=4.1 \mathrm{~Hz}, 1 \mathrm{H}, 5-\mathrm{H}\right)$, $3.979\left(\mathrm{dd},{ }^{3} J_{6,7}=6.9 \mathrm{~Hz},{ }^{3} J_{6,5}=5.3 \mathrm{~Hz}, 1 \mathrm{H}, 6-\mathrm{H}\right), 3.814$ $\left(\mathrm{dd},{ }^{2} J_{8 \mathrm{a}, 8 \mathrm{~b}}=11.6 \mathrm{~Hz},{ }^{3} J_{8 \mathrm{a}, 7}=3.3 \mathrm{~Hz}, 1 \mathrm{H}, 8-\mathrm{Ha}\right), 3.789$ (ddd, $\left.{ }^{3} J_{7,6}=6.9 \mathrm{~Hz},{ }^{3} J_{7,8 \mathrm{~b}}=6.9 \mathrm{~Hz},{ }^{3} J_{7,8 \mathrm{a}}=3.3 \mathrm{~Hz}, 1 \mathrm{H}, 7-\mathrm{H}\right), 3.677$ $\left(\mathrm{dd},{ }^{2} J_{8 \mathrm{~b}, 8 \mathrm{a}}=11.6 \mathrm{~Hz},{ }^{3} J_{8 \mathrm{~b}, 7}=6.9 \mathrm{~Hz}, 1 \mathrm{H}, 8-\mathrm{Hb}\right), 3.597(\mathrm{~d}$, $\left.{ }^{2} J_{1 \mathrm{a}, 1 \mathrm{~b}}=11.8 \mathrm{~Hz}, 1 \mathrm{H}, 1-\mathrm{Ha}\right), 3.589\left(\mathrm{~d},{ }^{2} J_{1 \mathrm{~b}, 1 \mathrm{a}}=11.8 \mathrm{~Hz}\right.$, $1 \mathrm{H}, 1-\mathrm{Hb}), 2.417\left(\mathrm{dd},{ }^{2} J_{3 \mathrm{a}, 3 \mathrm{~b}}=14.3 \mathrm{~Hz},{ }^{3} J_{3 \mathrm{a}, 4}=5.6 \mathrm{~Hz}\right.$, $1 \mathrm{H}, 3-\mathrm{Ha}), 2.055\left(\mathrm{dd},{ }^{2} J_{3 \mathrm{~b}, 3 \mathrm{a}}=14.3 \mathrm{~Hz},{ }^{3} J_{3 \mathrm{~b}, 4}=1.9 \mathrm{~Hz}\right.$, $1 \mathrm{H}, 3-\mathrm{Hb}) \mathrm{ppm} ;{ }^{13} \mathrm{C} \mathrm{NMR}\left(150.93 \mathrm{MHz}, \mathrm{D}_{2} \mathrm{O}, 25{ }^{\circ} \mathrm{C}\right): \delta$ $(\beta$-pyranose $)=97.25$ (2-C), 69.97 (7-C), 68.09 (1-C), 67.98 (4-C), 67.15 (6-C), 66.24 (5-C), 63.57 (8-C), 30.70 (3-C) $\mathrm{ppm} ; \delta$ (keto isomer) $=212.14(2-\mathrm{C}), 72.33(5-\mathrm{C}), 71.65$ (6-C), 71.64 (7-C), 69.36 (4-C), 68.55 (1-C), 63.35 (8-C), 42.15 (3-C) ppm; $\delta(\alpha$-furanose $)=105.37$ (2-C), 81.19 (5-C), 72.52 (4-C), 72.24 (7-C), 71.18 (6-C), 66.09 (1-C), 62.97 (8-C), 44.01 (3-C) ppm; $\delta(\beta$-furanose $)=105.96$ (2-C), 83.67 (5-C), 72.56 (4-C), 72.28 (7-C), 71.58 (6-C), 65.73 (1-C), 62.97 (8-C), 42.58 (3-C) ppm; HRMS (ESI ${ }^{+}$): $m / z=247.0791\left([\mathrm{M}+\mathrm{Na}]^{+}\right)$, calcd. For $\mathrm{C}_{9} \mathrm{H}_{18} \mathrm{O}_{6} \mathrm{Na}^{+}$ $247.0788 ;=[\alpha]_{D}^{20} 0.3\left(c=1.0\right.$, water); $R_{f}=0.5$ (1-butanol/ acetone/water $(5: 4: 1)$.

1,4,5,6,7,8,9-Hepta-O-acetyl-2,3-dideoxy-2-C-methylidene-D-glycero-D-galacto-nonitol (syn-25, $\mathrm{C}_{24} \mathrm{H}_{34} \mathrm{O}_{14}$ ) D-Mannose (200 mg, 19, $1.11 \mathrm{mmol}$ ) and $251 \mathrm{mg}$ 2-(bromomethyl)prop-2-en-1-ol (14, $1.67 \mathrm{mmol}, 1.5$ equiv.) were dissolved in $8 \mathrm{~cm}^{3}$ ethanol and $2 \mathrm{~cm}^{3}$ water. Indium powder (191 mg, $1.67 \mathrm{mmol}, 1.5$ equiv.) was added and the 
suspension was sonicated at $45{ }^{\circ} \mathrm{C}$ for $18 \mathrm{~h}$. The reaction mixture was cooled to room temperature and the $\mathrm{pH}$ was adjusted to 7 with $1 \mathrm{M}$ aqueous sodium hydroxide solution. The suspension was filtered over a plug of celite which was subsequently rinsed with $30 \mathrm{~cm}^{3}$ of methanol. The filtrate was concentrated under reduced pressure and the residue was dissolved in $6 \mathrm{~cm}^{3}$ pyridine and $6 \mathrm{~cm}^{3}$ acetic anhydride. The mixture was cooled with an ice bath, treated with $7 \mathrm{mg}$ 4-(dimethylamino)pyridine ( $0.06 \mathrm{mmol}, 0.5$ equiv.) and stirred overnight. The mixture was concentrated and the addition of toluene facilitated the process by forming a homogenous pressure-maximum azeotrope with pyridine [34]. The residue was taken up in $20 \mathrm{~cm}^{3}$ ethyl acetate and was washed with water $\left(3 \times 10 \mathrm{~cm}^{3}\right)$ and $10 \mathrm{~cm}^{3}$ brine. The organic layer was dried over magnesium sulfate, filtered and concentrated. The epimeric mixture syn-/anti-25 could be partially separated from the per- $O$-acetylated starting material by performing column chromatography using heptane/ ethyl acetate (10/1 v/v) as eluent. However, the respective epimers could not be isolated. The conversion of $60 \%$ (542 mg mixture with 1,2,3,4,6-penta- $O$-acetyl-D-mannose, $0.672 \mathrm{mmol}$ of epimeric products) over two steps and the diastereoselective excess of $69 \%$ could be determined by means of NMR.

Ethyl 2-(benzyloxymethyl)acrylate (29, $\mathrm{C}_{13} \mathrm{H}_{16} \mathrm{O}_{3}$ ) A suspension of $386 \mathrm{mg}$ sodium hydride ( $60 \%$ dispersion, $9.65 \mathrm{mmol}, 0.5$ equiv.) in $30 \mathrm{~cm}^{3}$ anhydrous diethyl ether under argon was cooled to $0{ }^{\circ} \mathrm{C}$. Benzyl alcohol $\left(4.0 \mathrm{~cm}^{3}\right.$, 39 mmol, 2 equiv.) was slowly added and the reaction mixture was stirred for $30 \mathrm{~min}$ at $0{ }^{\circ} \mathrm{C}$. Subsequently, $3.9 \mathrm{~cm}^{3}$ trichloroacetonitrile ( $39 \mathrm{mmol}, 2$ equiv.) was slowly added and the solution was allowed to stir at room temperature for three hours. The reaction was stopped by the addition of 30 $\mathrm{cm}^{3}$ saturated aqueous bicarbonate solution. The aqueous phase was extracted with diethyl ether $\left(3 \times 30 \mathrm{~cm}^{3}\right)$ and the combined organic layers were dried over magnesium sulfate. After filtration, the eluate was concentrated to give crude benzyl 2,2,2-trichloroacetminidate (28) in form of a brown oil. To a mixture of $2.51 \mathrm{~g}$ ethyl 2-(hydroxymethyl)acrylate $(27,19.3 \mathrm{mmol})$ in $50 \mathrm{~cm}^{3}$ anhydrous diethyl ether under argon, $170 \mathrm{~mm}^{3}$ trifluoromethanesulfonic acid (1.92 mmol, 0.1 equiv.) was added followed by freshly prepared crude benzyl 2,2,2-trichloroacetimidate (28, 39 mmol, 2 equiv.). The solution was stirred for $42 \mathrm{~h}$ at room temperature. After complete consumption of the starting material, $30 \mathrm{~cm}^{3}$ aqueous hydrochloric acid solution ( $2 \mathrm{M}$ ) was added and the mixture was stirred for $10 \mathrm{~min}$. The organic phase was separated and washed with saturated bicarbonate solution $\left(2 \times 30 \mathrm{~cm}^{3}\right)$ followed by $30 \mathrm{~cm}^{3}$ of brine. After concentration, $50 \mathrm{~cm}^{3}$ heptane was added to the resulted white precipitate and the suspension was filtered and washed with $30 \mathrm{~cm}^{3}$ of heptane. The organic solution was dried over magnesium sulfate, filtered, and concentrated. The residue was purified by column chromatography using heptane/ethyl acetate (19/1 14/1 v/v) as eluent to give $3.70 \mathrm{~g}$ ethyl 2-(benzyloxymethyl)acrylate $(\mathbf{2 9}, 16.8 \mathrm{mmol}, 87 \%)$ as a yellowish liquid. ${ }^{1} \mathrm{H} \mathrm{NMR}\left(600.25 \mathrm{MHz}, \mathrm{CDCl}_{3}, 25{ }^{\circ} \mathrm{C}\right): \delta=7.407-$ 7.326 (m, 4H, H-7, H-8), 7.326-7.270 (m, 1H, H-9), 6.324 $\left(\mathrm{dd},{ }^{3} J_{4 \mathrm{a}, 3}=1.5 \mathrm{~Hz},{ }^{2} J_{4 \mathrm{a}, 4 \mathrm{~b}}=1.4 \mathrm{~Hz}, 1 \mathrm{H}, \mathrm{H}-4 \mathrm{a}\right), 5.927$ (dd, $\left.{ }^{3} J_{4 \mathrm{~b}, 3}=1.9 \mathrm{~Hz},{ }^{2} J_{4 \mathrm{~b}, 4 \mathrm{a}}=1.4 \mathrm{~Hz}, 1 \mathrm{H}, \mathrm{H}-4 \mathrm{a}\right), 4.594(\mathrm{~s}, 2 \mathrm{H}$, H-5), 4.248 (dd, $\left.{ }^{3} J_{3,4 \mathrm{~b}}=1.9 \mathrm{~Hz},{ }^{3} J_{3,4 \mathrm{a}}=1.5 \mathrm{~Hz}, 2 \mathrm{H}, \mathrm{H}-3\right)$, $4.226\left(\mathrm{q},{ }^{3} J_{1^{\prime}, 2^{\prime}}=7.2 \mathrm{~Hz}, 2 \mathrm{H}, \mathrm{H}-1^{\prime}\right), 1.304\left(\mathrm{t},{ }^{3} J_{2^{\prime}, 1^{\prime}}=7.2 \mathrm{~Hz}\right.$, $\left.3 \mathrm{H}, \mathrm{H}-2^{\prime}\right) \mathrm{ppm} ;{ }^{13} \mathrm{C} \mathrm{NMR}\left(150.93 \mathrm{MHz}, \mathrm{CDCl}_{3}, 25{ }^{\circ} \mathrm{C}\right)$ : $\delta=166.04(\mathrm{C}-1), 138.23$ (C-6), 137.57 (C-2), 128.56 (C-8), 127.82 (C-9), 127.77 (C-7), 125.81 (C-4), 72.89 (C-5), 68.52 (C-3), 60.85 (C-1'), 14.34 (C-2') ppm; HRMS $\left(\mathrm{ESI}^{+}\right): m / z=243.0992\left([\mathrm{M}+\mathrm{Na}]^{+}\right)$, calcd. for $\mathrm{C}_{13} \mathrm{H}_{16} \mathrm{NaO}_{3}{ }^{+}$ 243.0992; $R_{f}=0.5$ (heptane/ethyl acetate $=4: 1$ ).

2- (Benzyloxymethyl)-prop-2-en-1-ol (30, $\mathbf{C}_{\mathbf{1 1}} \mathbf{H}_{\mathbf{1 4}} \mathbf{O}_{\mathbf{2}}$ ) Ethyl 2-(benzyloxymethyl)acrylate (29, 3.70 g, $16.8 \mathrm{mmol}$ ) was dissolved in anhydrous dichloromethane and cooled to $-78{ }^{\circ} \mathrm{C}$. DIBAL-H solution $\left(39 \mathrm{~cm}^{3}, 1 \mathrm{M}\right.$ in dichloromethane, $39 \mathrm{mmol}, 2.3$ equiv.) was slowly added over $30 \mathrm{~min}$. After stirring for an hour at $-78^{\circ} \mathrm{C}$, the solution was allowed to warm to $0{ }^{\circ} \mathrm{C}$ using an ice bath. The reaction was stopped by slowly adding $75 \mathrm{~cm}^{3}$ of a saturated potassium sodium tartrate solution. The ice bath was removed and the mixture was stirred overnight at room temperature. The organic layer was separated and the aqueous phase was extracted with dichloromethane $\left(3 \times 50 \mathrm{~cm}^{3}\right)$. The combined organic layers were washed with $50 \mathrm{~cm}^{3}$ brine, dried over magnesium sulfate, filtered, and concentrated. The residue was purified by column chromatography using heptane/ethyl acetate (4/1 v/v) as eluent to give $2.21 \mathrm{~g}$ 2-(benzyloxymethyl)-prop-2-en-1-ol (30, $12.4 \mathrm{mmol}, 74 \%)$ as a yellowish oil. ${ }^{1} \mathrm{H}$ NMR $\left(600.25 \mathrm{MHz} \mathrm{CDCl}_{3}, 25^{\circ} \mathrm{C}\right)$ : $\delta=7.400-7.319(\mathrm{~m}, 4 \mathrm{H}, \mathrm{H}-7, \mathrm{H}-8), 7.319-7.272(\mathrm{~m}, 1 \mathrm{H}$, H-9), $5.211\left(\mathrm{dd},{ }^{2} J_{4 \mathrm{a}, 4 \mathrm{~b}}=1.7 \mathrm{~Hz},{ }^{3} J_{4 \mathrm{a}, 3}=0.9 \mathrm{~Hz}, 1 \mathrm{H}, \mathrm{H}-4 \mathrm{a}\right)$, $5.161\left(\mathrm{dd},{ }^{2} J_{4 \mathrm{~b}, 4 \mathrm{a}}=1.7 \mathrm{~Hz},{ }^{3} J_{4 \mathrm{~b}, 3}=1.0 \mathrm{~Hz}, 1 \mathrm{H}, \mathrm{H}-4 \mathrm{~b}\right), 4.528$ (s, 2H, H-5), 4.206 (d, $\left.{ }^{3} J_{1, \mathrm{OH}}=5.4 \mathrm{~Hz}, 2 \mathrm{H}, \mathrm{H}-1\right), 4.106(\mathrm{dd}$, $\left.{ }^{3} J_{3,4 \mathrm{~b}}=1.0 \mathrm{~Hz},{ }^{3} J_{3,4 \mathrm{a}}=0.9 \mathrm{~Hz}, 2 \mathrm{H}, \mathrm{H}-3\right), 1.895(\mathrm{~m}, 1 \mathrm{H}, \mathrm{OH})$ ppm; ${ }^{13} \mathrm{C} \mathrm{NMR}\left(150.93 \mathrm{MHz}, \mathrm{CDCl}_{3}, 25{ }^{\circ} \mathrm{C}\right): \delta=145.14$ (C-2), 138.08 (C-6), 128.60-127.90 (C-7, C-8, C-9), 113.75 (C-4), 72.50 (C-5), 72.00 (C-3), 64.81 (C-1) ppm; HRMS $\left(\mathrm{ESI}^{+}\right): m / z=201.0887\left([\mathrm{M}+\mathrm{Na}]^{+}\right)$, calcd. for $\mathrm{C}_{11} \mathrm{H}_{14} \mathrm{NaO}_{2}{ }^{+}$ 201.0886; $R_{f}=0.5$ (heptane/ethyl acetate $=1: 1$ ).

2-(Benzyloxymethyl)allyl bromide $\left(16, \mathrm{C}_{11} \mathrm{H}_{13} \mathrm{BrO}\right)$ Triphenylphospine (3.47 g, $13.2 \mathrm{mmol}, 2$ equiv.) and $4.39 \mathrm{~g}$ tetrabromomethane (13.24 mmol, 2 equiv.) were dissolved in $150 \mathrm{~cm}^{3}$ of anhydrous dichloromethane under argon, cooled to $0{ }^{\circ} \mathrm{C}$ and stirred for $15 \mathrm{~min}$. Subsequently, a solution of $1.18 \mathrm{~g}$ compound $30(6.62 \mathrm{mmol})$ in $15 \mathrm{~cm}^{3}$ anhydrous dichloromethane was added. The reaction was monitored by 
TLC and was completed after $3 \mathrm{~h}$. After concentration, the residue was dissolved in $50 \mathrm{~cm}^{3}$ diethyl ether, and $50 \mathrm{~cm}^{3}$ of heptane was added under stirring. The resulting precipitate was filtered over celite and washed with $10 \mathrm{~cm}^{3}$ heptane. The eluate was washed with water $\left(3 \times 50 \mathrm{~cm}^{3}\right)$ and $50 \mathrm{~cm}^{3}$ brine, dried over magnesium sulfate, filtered, and concentrated. The residue was purified by column chromatography using heptane/ethyl acetate $(19 / 1 \mathrm{v} / \mathrm{v})$ as eluent to give $1.24 \mathrm{~g}$ 2-(benzyloxymethyl)allyl bromide (16, $5.14 \mathrm{mmol}$, $78 \%)$ as colorless liquid. ${ }^{1} \mathrm{H}$ NMR $\left(600.25 \mathrm{MHz}, \mathrm{CDCl}_{3}\right.$, $\left.25^{\circ} \mathrm{C}\right): \delta=7.511-7.326(\mathrm{~m}, 4 \mathrm{H}, \mathrm{H}-7, \mathrm{H}-8), 7.326-7.272$ (m, $1 \mathrm{H}, \mathrm{H}-9), 5.359$ (ddd, ${ }^{2} J_{4 \mathrm{a}, 4 \mathrm{~b}}=1.2 \mathrm{~Hz},{ }^{3} J_{4 \mathrm{a}, 3}=0.8 \mathrm{~Hz}$, $\left.{ }^{3} J_{4 \mathrm{a}, 1}=0.8 \mathrm{~Hz}, 1 \mathrm{H}, \mathrm{H}-4 \mathrm{a}\right), 5.271\left(\mathrm{ddd},{ }^{3} J_{4 \mathrm{~b}, 3}=1.3 \mathrm{~Hz}\right.$, $\left.{ }^{2} J_{4 \mathrm{~b}, 4 \mathrm{a}}=1.2 \mathrm{~Hz},{ }^{3} J_{4 \mathrm{~b}, 1}=0.2 \mathrm{~Hz}, 1 \mathrm{H}, \mathrm{H}-4 \mathrm{~b}\right), 4.537(\mathrm{~s}, 2 \mathrm{H}$, $\mathrm{H}-5), 4.156\left(\mathrm{dd},{ }^{3} J_{3,4 \mathrm{~b}}=1.3 \mathrm{~Hz},{ }^{3} J_{4 \mathrm{a}, 3}=0.8 \mathrm{~Hz}, 2 \mathrm{H}, \mathrm{H}-3\right)$, $4.053\left(\mathrm{dd},{ }^{3} J_{1,4 \mathrm{a}}=0.8 \mathrm{~Hz},{ }^{3} J_{1,4 \mathrm{~b}}=0.2 \mathrm{~Hz}, 2 \mathrm{H}, \mathrm{H}-1\right) \mathrm{ppm}$; ${ }^{13} \mathrm{C}$ NMR $\left(150.93 \mathrm{MHz}, \mathrm{CDCl}_{3}, 25{ }^{\circ} \mathrm{C}\right): \delta=142.51(\mathrm{C}-2)$, 138.14 (C-6), 128.58-127.88 (C-7, C-8, C-9), 117.44 (C-4), 72.59 (C-5), 70.49 (C-3), 33.21 (C-1) ppm; HRMS (ESI ${ }^{+}$): $m / z=263.0038\left([\mathrm{M}+\mathrm{Na}]^{+}\right)$, calcd. for $\mathrm{C}_{11} \mathrm{H}_{13} \mathrm{BrNaO}^{+}$ 263.0042; $R_{f}=0.5$ (heptane/ethyl acetate $=14: 1$ ).

$4,5,6,7,8,9-\mathrm{H}$ ex a - O- a c e t y l- 1 - O- b e n zyl-2,3-dideoxy-2-C-methylidene-D-glycero-D-gaIacto-nonitol (syn-31, $\mathrm{C}_{\mathbf{2}} \mathrm{H}_{\mathbf{3 8}} \mathrm{O}_{13}$ ) D-Mannose (19, $200 \mathrm{mg}, 1.11 \mathrm{mmol}$ ) and $401 \mathrm{mg}$ 2-(benzyloxymethyl) allyl bromide (16, $1.67 \mathrm{mmol}, 1.5$ equiv.) were dissolved in $8 \mathrm{~cm}^{3}$ ethanol and $2 \mathrm{~cm}^{3}$ water. Indium powder $(191 \mathrm{mg}$, $1.67 \mathrm{mmol}, 1.5$ equiv.) was added and the suspension was sonicated at $45^{\circ} \mathrm{C}$ for $18 \mathrm{~h}$. The reaction mixture was cooled to room temperature and the $\mathrm{pH}$ was adjusted to 7 with $1 \mathrm{M}$ aqueous sodium hydroxide solution. The suspension was filtered over a plug of celite which was subsequently rinsed with $30 \mathrm{~cm}^{3}$ of methanol. The filtrate was concentrated and the residue was dissolved in $6 \mathrm{~cm}$ pyridine and $6 \mathrm{~cm}^{3}$ acetic anhydride. The mixture was cooled with an ice bath, treated with $7 \mathrm{mg} \mathrm{4-(dimethylamino)pyridine}(0.06 \mathrm{mmol}, 0.5$ equiv.) and stirred overnight. The reaction mixture was concentrated and the addition of toluene facilitated the process by forming a homogenous pressure-maximum azeotrope with pyridine [34]. The residue was taken up in $20 \mathrm{~cm}^{3}$ ethyl acetate and was washed with water $\left(3 \times 10 \mathrm{~cm}^{3}\right)$ and $10 \mathrm{~cm}^{3}$ brine. The organic layer was dried over magnesium sulfate, filtered, and concentrated. The epimeric mixture could be separated from the per- $O$-acetylated starting material by performing column chromatography using heptane/ethyl acetate $(2 / 1 \mathrm{v} / \mathrm{v})$ as eluent. The epimers $\boldsymbol{s y n - 3 1}$ and $\boldsymbol{a n t i - 3 1}$ could not be separated by conventional column chromatography but by HPLC using a Macherey-Nagel NUCLEODUR ${ }^{\circledR}$ 100-5 column. For successful separation, the column was cooled to $15{ }^{\circ} \mathrm{C}$. The analytes were detected at $254 \mathrm{~nm}$ and the eluent applied consisted of heptane/ethyl acetate $=86 / 14$. The conversion of $45 \%$ ( $298 \mathrm{mg}, 0.501 \mathrm{mmol}$ ) over two steps and the diastereoselective excess of $60 \%$ could be determined by means of NMR. HRMS $\left(\mathrm{ESI}^{+}\right): \mathrm{m} / z=617.2209$ $\left([\mathrm{M}+\mathrm{Na}]^{+}\right)$, calcd. for $\mathrm{C}_{29} \mathrm{H}_{38} \mathrm{NaO}_{13}{ }^{+}$617.2205; $R_{f}=0.5$ (heptane/ethyl acetate $=1: 1$ ).

syn-31 : ${ }^{1} \mathrm{H}$ NMR $\left(600.25 \mathrm{MHz}, \mathrm{CDCl}_{3}, 25^{\circ} \mathrm{C}\right): \delta=7.400$ 7.309 (m, 4H, H-3", H-4"), 7.309-7.266 (m, 1H, H-5"), $5.433\left(\mathrm{dd},{ }^{3} J_{6,5}=10.0 \mathrm{~Hz},{ }^{3} J_{6,7}=2.0 \mathrm{~Hz}, 1 \mathrm{H}, \mathrm{H}-6\right), 5.316$ $\left(\mathrm{dd},{ }^{3} J_{7,8}=8.6 \mathrm{~Hz},{ }^{3} J_{7,6}=1.97 \mathrm{~Hz}, 1 \mathrm{H}, \mathrm{H}-7\right), 5.189(\mathrm{dd}$, $\left.{ }^{3} J_{5,6}=10.0 \mathrm{~Hz},{ }^{3} J_{5,4}=2.0 \mathrm{~Hz}, 1 \mathrm{H}, \mathrm{H}-5\right), 5.127(\mathrm{ddd}$, $\left.{ }^{3} J_{4,3 \mathrm{~b}}=9.3 \mathrm{~Hz},{ }^{3} J_{4,3 \mathrm{a}}=4.0 \mathrm{~Hz},{ }^{3} J_{4,5}=2.0 \mathrm{~Hz}, 1 \mathrm{H}, \mathrm{H}-4\right), 5.071$ (m, 1H, H-1"a), 4.981 (ddd, ${ }^{3} J_{8,7}=8.6 \mathrm{~Hz},{ }^{3} J_{8,9 \mathrm{~b}}=5.4 \mathrm{~Hz}$, $\left.{ }^{3} J_{8,9 \mathrm{a}}=2.9 \mathrm{~Hz}, 1 \mathrm{H}, \mathrm{H}-8\right), 4.955$ (s, 1H, H-1"b), $4.506(\mathrm{~d}$, $\left.{ }^{2} J_{1^{\prime} \mathrm{a}, 1^{\prime} \mathrm{b}}=11.9 \mathrm{~Hz}, 1 \mathrm{H}, \mathrm{H}-1^{\prime} \mathrm{a}\right), 4.406\left(\mathrm{~d},{ }^{2} J_{1^{\prime} \mathrm{b}, 1^{\prime} \mathrm{a}}=11.9 \mathrm{~Hz}\right.$, $1 \mathrm{H}, \mathrm{H}-1$ 'b), $4.211\left(\mathrm{dd},{ }^{2} J_{9 \mathrm{a}, 9 \mathrm{~b}}=12.5 \mathrm{~Hz},{ }^{3} J_{9 \mathrm{a}, 8}=2.9 \mathrm{~Hz}\right.$, 1H, H-9a), 4.095 (d, $\left.{ }^{2} J_{1 \mathrm{a}, 1 \mathrm{~b}}=12.75 \mathrm{~Hz}, 1 \mathrm{H}, \mathrm{H}-1 \mathrm{a}\right), 4.021$ $\left(\mathrm{dd},{ }^{2} J_{9 \mathrm{~b}, 9 \mathrm{a}}=12.5 \mathrm{~Hz},{ }^{3} J_{9 \mathrm{~b}, 8}=5.4 \mathrm{~Hz}, 1 \mathrm{H}, \mathrm{H}-9 \mathrm{~b}\right), 3.886$ (d, $\left.{ }^{2} J_{1 \mathrm{~b}, 1 \mathrm{a}}=12.75 \mathrm{~Hz}, 1 \mathrm{H}, \mathrm{H}-1 \mathrm{~b}\right), 2.328\left(\mathrm{dd},{ }^{2} J_{3 \mathrm{a}, 3 \mathrm{~b}}=14.2 \mathrm{~Hz}\right.$, $\left.{ }^{3} J_{3 \mathrm{a}, 4}=4.0 \mathrm{~Hz}, 1 \mathrm{H}, \mathrm{H}-3 \mathrm{a}\right), 2.126\left(\mathrm{dd},{ }^{2} J_{3 \mathrm{~b}, 3 \mathrm{a}}=14.2 \mathrm{~Hz}\right.$, ${ }^{3} J_{3 \mathrm{~b}, 4}=9.3 \mathrm{~Hz}, 1 \mathrm{H}, \mathrm{H}-3 \mathrm{~b}$ ), 2.127 (s, 3H, OAc), 2.085 (s, 3H, OAc), 2.058 (s, 3H, OAc), 2.035 (s, 3H, OAc), 2.007 (s, $3 \mathrm{H}, \mathrm{OAc}), 1.994$ (s, 3H, OAc) ppm; ${ }^{13} \mathrm{C}$ NMR $(150.93 \mathrm{MHz}$, $\left.\mathrm{CDCl}_{3}, 25^{\circ} \mathrm{C}\right): \delta=170.69\left((\underline{\mathrm{C}}=\mathrm{O})-\mathrm{CH}_{3}\right), 170.39((\mathrm{C}=\mathrm{O})-$ $\left.\mathrm{CH}_{3}\right), 170.16\left((\underline{\mathrm{C}}=\mathrm{O})-\mathrm{CH}_{3}\right), 170.10\left((\underline{\mathrm{C}}=\mathrm{O})-\mathrm{CH}_{3}\right), 170.09$ $\left((\underline{\mathrm{C}}=\mathrm{O})-\mathrm{CH}_{3}\right), 169.81\left((\underline{\mathrm{C}}=\mathrm{O})-\mathrm{CH}_{3}\right), 141.16(\mathrm{C}-2), 138.41$ (C-2"), $128.52\left(\mathrm{C}-4^{\prime \prime}\right), 127.89\left(\mathrm{C}-3^{\prime \prime}\right), 127.75\left(\mathrm{C}-5^{\prime \prime}\right), 116.23$ (C-1"), 72.46 (C-1), 71.97 (C-1'), 69.67 (C-5), 68.35 (C-8), 68.08 (C-4), 67.65 (C-7), 67.18 (C-6), 62.06 (C-9), 35.32 (C-3), $21.08\left((\mathrm{C}=\mathrm{O})-\underline{\mathrm{CH}}_{3}\right), 20.98\left((\mathrm{C}=\mathrm{O})-\mathrm{CH}_{3}\right), 20.93$ $\left((\mathrm{C}=\mathrm{O})-\underline{\mathrm{CH}}_{3}\right), 20.85\left((\mathrm{C}=\mathrm{O})-\underline{\mathrm{C}} \mathrm{H}_{3}\right), 20.83\left((\mathrm{C}=\mathrm{O})-\underline{\mathrm{C}} \mathrm{H}_{3}\right)$, $20.75\left((\mathrm{C}=\mathrm{O})-\mathrm{CH}_{3}\right) \mathrm{ppm}$.

anti-31: ${ }^{1} \mathrm{H} \mathrm{NMR}\left(600.25 \mathrm{MHz}, \mathrm{CDCl}_{3}, 25^{\circ} \mathrm{C}\right): \delta=7.400$ 7.309 (m, 4H, H-3", H-4"), 7.309-7.266 (m, 1H, H-5"), $5.411\left(\mathrm{dd},{ }^{3} J_{6,5}=9.4 \mathrm{~Hz},{ }^{3} J_{6,7}=2.2 \mathrm{~Hz}, 1 \mathrm{H}, \mathrm{H}-6\right), 5.371$ $\left(\mathrm{dd},{ }^{3} J_{7,8}=9.2 \mathrm{~Hz},{ }^{3} J_{7,6}=2.2 \mathrm{~Hz}, 1 \mathrm{H}, \mathrm{H}-7\right), 5.202(\mathrm{dd}$, $\left.{ }^{3} J_{5,6}=9.4 \mathrm{~Hz},{ }^{3} J_{5,4}=2.2 \mathrm{~Hz}, 1 \mathrm{H}, \mathrm{H}-5\right), 5.118\left(\mathrm{~s}, 1 \mathrm{H}, \mathrm{H}-1{ }^{\prime \prime} \mathrm{a}\right)$, $5.067\left(\mathrm{ddd},{ }^{3} J_{4,3 \mathrm{a}}=10.8 \mathrm{~Hz},{ }^{3} J_{4,5}=2.2 \mathrm{~Hz},{ }^{3} J_{4,3 \mathrm{~b}}=0.5 \mathrm{~Hz}\right.$, $1 \mathrm{H}, \mathrm{H}-4), 5.051\left(\mathrm{ddd},{ }^{3} J_{8,7}=9.2 \mathrm{~Hz},{ }^{3} J_{8,9 \mathrm{~b}}=5.4 \mathrm{~Hz}\right.$, $\left.{ }^{3} J_{8,9 \mathrm{a}}=2.8 \mathrm{~Hz}, 1 \mathrm{H}, \mathrm{H}-8\right), 4.955$ (s, 1H, H-1" $\left.{ }^{\prime} \mathrm{b}\right), 4.544$ (d, $\left.{ }^{2} J_{1^{\prime} \mathrm{a}, 1^{\prime} \mathrm{b}}=12.0 \mathrm{~Hz}, 1 \mathrm{H}, \mathrm{H}-1^{\prime} \mathrm{a}\right), 4.481\left(\mathrm{~d},{ }^{2} J_{1^{\prime} \mathrm{b}, 1^{\prime} \mathrm{a}}=12.0 \mathrm{~Hz}\right.$, $1 \mathrm{H}, \mathrm{H}-1$ 'b), $4.203\left(\mathrm{dd},{ }^{2} J_{9 \mathrm{a}, 9 \mathrm{~b}}=12.5 \mathrm{~Hz},{ }^{3} J_{9 \mathrm{a}, 8}=2.8 \mathrm{~Hz}\right.$, $1 \mathrm{H}, \mathrm{H}-9 \mathrm{a}), 4.036\left(\mathrm{dd},{ }^{2} J_{9 \mathrm{~b}, 9 \mathrm{a}}=12.5 \mathrm{~Hz},{ }^{3} J_{9 \mathrm{~b}, 8}=5.4 \mathrm{~Hz}, 1 \mathrm{H}\right.$, $\mathrm{H}-9 \mathrm{~b}), 3.945$ (d, $\left.{ }^{2} J_{1 \mathrm{a}, 1 \mathrm{~b}}=12.7 \mathrm{~Hz}, 1 \mathrm{H}, \mathrm{H}-1 \mathrm{a}\right), 3.907$ (d, $\left.{ }^{2} J_{1 \mathrm{~b}, 1 \mathrm{a}}=12.7 \mathrm{~Hz}, 1 \mathrm{H}, \mathrm{H}-1 \mathrm{~b}\right), 2.518\left(\mathrm{dd},{ }^{2} J_{3 \mathrm{a}, 3 \mathrm{~b}}=15.1 \mathrm{~Hz}\right.$, $\left.{ }^{3} J_{3 \mathrm{a}, 4}=10.8 \mathrm{~Hz}, 1 \mathrm{H}, \mathrm{H}-3 \mathrm{a}\right), 2.349\left(\mathrm{dd},{ }^{2} J_{3 \mathrm{~b}, 3 \mathrm{a}}=15.1 \mathrm{~Hz}\right.$, ${ }^{3} J_{3 \mathrm{~b}, 4}=0.5 \mathrm{~Hz}, 1 \mathrm{H}, \mathrm{H}-3 \mathrm{~b}$ ), 2.079 (s, 3H, OAc), 2.062 (s, $6 \mathrm{H}, 2 \mathrm{xOAc}$ ), 2.050 (s, 3H, OAc), 2.046 (s, 3H, OAc), 1.957 (s, 3H, OAc) ppm; ${ }^{13} \mathrm{C} \mathrm{NMR}\left(150.93 \mathrm{MHz}, \mathrm{CDCl}_{3}, 25^{\circ} \mathrm{C}\right)$ : $\delta=170.41-169.89\left(6 \mathrm{x}\left((\underline{\mathrm{C}}=\mathrm{O})-\mathrm{CH}_{3}\right)\right), 141.38(\mathrm{C}-2), 138.46$ (C-2"), 128.48 (C-4"), $127.83\left(\mathrm{C}-3^{\prime \prime}\right), 127.70\left(\mathrm{C}-5^{\prime \prime}\right), 115.10$ (C-1"), 73.07 (C-1), 72.02 (C-1'), 70.82 (C-4), 69.82 (C-5), 
68.06 (C-8), 67.82 (C-7), 67.59 (C-6), 61.99 (C-9), 31.89 (C-3), 21.08-20.75 (6x $\left.(\mathrm{C}=\mathrm{O})-\mathrm{CH}_{3}\right) \mathrm{ppm}$.

Acknowledgements Open access funding provided by University of Vienna. We would like to thank the NMR centre for NMR analysis, the mass spectrometry centre for mass analysis and A. Roller for X-ray crystallographic analysis. We also thank T. Wrodnigg and J. Schörghuber for critical comments on the manuscript.

Open Access This article is distributed under the terms of the Creative Commons Attribution 4.0 International License (http://creativeco mmons.org/licenses/by/4.0/), which permits unrestricted use, distribution, and reproduction in any medium, provided you give appropriate credit to the original author(s) and the source, provide a link to the Creative Commons license, and indicate if changes were made.

\section{References}

1. Li CJ, Chan TH (1999) Tetrahedron 55:11149

2. Paquette LA (2003) Synthesis 5:765

3. Podlech J, Maier TC (2003) Synthesis 5:633

4. Bowyer WJ, Singaram B, Sessler AM (2011) Tetrahedron 67:7449

5. Albler C, Hollaus R, Kählig H (2014) Schmid W 10:2230

6. Binder WH, Prenner RH, Schmid W (1994) Tetrahedron 3:749

7. Prenner RH, Binder WH, Schmid W (1994) Eur J Org Chem 1:73

8. Li CJ (2005) Chem Rev 105:3095

9. Kim E, Gordon DM, Schmid W, Whitesides GM (1993) J Org Chem 58:5500

10. Gordon DM, Whitesides GM (1993) J Org Chem 58:7937

11. Chan TH, Lee MC (1995) J Org Chem 60:4228

12. Chan TH, Li C (1992) J Chem Soc Chem Commun 10:747

13. Reiner M, Schmidt RR (2000) Tetrahedron: Asymmetry 11:319

14. Gintner M, Yondea Y, Schmölzer C, Denner C, Kählig H, Schmid W (2019) Carb Res 474:34

15. Matthies S, Stallforth P, Seeberger PH (2015) J Am Chem Soc 137:2848

16. Prenner RH, Schmid W (1996) Monatsh Chem 127:1045

17. Knecht KJ, Feather MS, Baynes JW (1992) Arch Biochem Biophys 294:130
18. Kato H, van Chuyen N, Shinoda Y, Sekiya F, Hayase F (1990) Biochim Biophys Acta 1035:71

19. Wells-Knecht KJ, Lyons TJ, McCance DR, Thorpe SR, Feather MS, Baynes JW (1994) Diabetes 43:1152

20. Yamada H, Miyata S, Igaki N, Yatabe H, Miyauchi Y, Ohara T, Sakai M, Shoda H, Oimomi M, Kasuga M (1994) J Biol Chem 269:20275

21. Shin DB, Hayase F, Kato H (1988) Agric Biol Chem 52:1451

22. Szwergold BS, Kappler F, Brown TR (1990) Science 247:451

23. Kotecha JA, Feather MS, Kubiseski TJ, Walton DJ (1996) Carb Res 289:77

24. Schmölzer C, Fischer M, Schmid W (2010) Eur J Org Chem 25:4886

25. Villieras J, Rambaud M (1988) Org Synth 66:220

26. Komatsu Y, Watanabe R, Ikishima H, Nakano K, Ichikawa Y, Kotsuki H (2012) Org Biomol Chem 10:2993

27. De Alvarenga ES, Carneiro VMT, Silvério FO, Saliba WA (2006) J Chil Chem Soc 51:986

28. Sugisaki CH, Ruland Y, Baltas M (2003) Eur J Org Chem 4:672

29. Trappeniers M, Goormans S, Van Beneden K, Decruy T, Linclau B, Al-Shamkhani A, Elliott T, Ottensmeier C, Werner JM, Elewaut D, Van Calenbergh S (2008) ChemMedChem 3:1061

30. Schmidt RR, Kinzy W (1994) Adv Carbohydr Chem Biochem 50:21

31. Farhane S, Fournier MA, Maltais R, Poirier D (2011) Tetrahedron 13:2434

32. Appel R (1975) Angew Chem Int Ed 14:801

33. Szarek WA, Rafka RJ, Yang TF, Martin OR (1995) Can J Chem 73:1639

34. Gmehling J, Menke J, Krafczyk J, Fischer K, Fontaine JC, Kehiaian HV (2011) Azeotropic data for binary mixtures. In: Haynes WM, Lide DR (eds) CRC Handbook of Chemistry and Physics, 92nd edn. CRC Press, Boca Raton, p 6

Publisher's Note Springer Nature remains neutral with regard to jurisdictional claims in published maps and institutional affiliations. 